\title{
Non-local stiffness and damping models for shear-deformable beams
}

\author{
Mario Di Paola ${ }^{a}$, Giuseppe Failla ${ }^{\mathrm{b}, *}$, Massimiliano Zingales ${ }^{\mathrm{a}, \mathrm{c}}$ \\ a Dipartimento di Ingegneria Civile, Ambientale e Aerospaziale (DICA), Università di Palermo, Viale delle Scienze, 90128, Italy \\ ${ }^{\mathrm{b}}$ Dipartimento di Ingegneria Civile, dell'Energia, dell'Ambiente e dei Materiali (DICEAM), Università Mediterranea di Reggio Calabria, Via Graziella, Localitá \\ Feo di Vito, \\ 89124 Reggio Calabria, Italy \\ ${ }^{\mathrm{c}}$ Istituto Euro-Mediterraneo di Scienza e Tecnologia (IEMEST), Via Emerico Amari 123, 90100 Palermo, Italy
}

\section{A R T I C L E I N F O}

\section{Article history:}

Received 25 May 2012

Accepted 26 December 2012

Available online 11 January 2013

\section{Keywords:}

Non-local elasticity

Non-local damping

Long-range interactions

Timoshenko beam

\begin{abstract}
A B S T R A C T
This paper presents the dynamics of a non-local Timoshenko beam. The key assumption involves modeling non-local effects as long-range volume forces and moments mutually exerted by non-adjacent beam segments, that contribute to the equilibrium of any beam segment along with the classical local stress resultants. Elastic and viscous long-range volume forces/moments are endowed in the model. They are built as linearly depending on the product of the volumes of the interacting beam segments and on generalized measures of their relative motion, based on the pure deformation modes of the beam. Attenuation functions governing the space decay of the non-local effects are introduced. Numerical results are presented for a variety of non-local parameters.
\end{abstract}

(c) 2013 Elsevier Masson SAS. All rights reserved.

\section{Introduction}

Since the very early studies on those phenomena where longrange intermolecular forces and microstructural effects play a crucial role, the inadequacies of the intrinsically free-scale local continuum approach and, on the other hand, the computational difficulties involved by a discrete modeling of a medium at an atomic scale, have encouraged the search for alternative and more effective theories. As a result of the efforts in this field, non-local theories have been formulated. Most of these rely on the idea that microstructural effects can be accounted in an average sense, by formulating an enriched continuum with non-local terms. Typical examples are the integral theory (Eringen, 1972, 1983, 1987), the gradient elasticity theory (Aifantis, 1984, 1999; Chang et al., 2002), the peridynamic theory (Silling, 2000; Silling et al., 2003) and the so-called continualization theories, where a discrete medium is translated into a non-local continuous model (Askes and Metrikine, 2002; Metrikine and Askes, 2002). A nonlocal 3D continuum has been recently proposed also by the authors, where long-range elementary volume forces are introduced, as resulting from the relative displacement between elementary volume elements (Di Paola et al., 2009, 2010a,b).

In the last two decades, non-local beam and plate engineering theories have been derived from non-local enriched 3D continua.

\footnotetext{
* Corresponding author. Tel.: +39 (0) 965 875221; fax: +39 (0) 965875201.

E-mail address: giuseppe.failla@unirc.it (G. Failla).
}

This interest has been certainly boosted by the increasing importance of nanotechnologies, where beam- and plate-like devices are employed as sensors and actuators for vibration damping, for instance in electromechanical systems (for a review see Qian et al., 2002). On the other hand, the importance of microstructural effects in micro- and nano-components has been shown by atomistic simulations (Wang and $\mathrm{Hu}, 2005$ ) and experimental evidence on several materials, such as polypropylene (McFarland and Colton, 2005), epoxy (Lam et al., 2003), graphite (Tang, 1983) and copper (Poole et al., 1996).

There exists a remarkable variety of non-local beam theories, especially for dynamic applications. In general a non-local linearly elastic stiffness has been considered, in context with the classical Euler-Bernoulli (EB) or the Timoshenko (TM) beam theory. Lu et al. (2007), Reddy (2007), Aydogdu (2009), Murmu and Pradhan (2009) have built non-local EB or TM beams where non-local effects are modeled by the Eringen integral theory, that involves a stressstrain relation between the stress at a given point and the strain in the whole beam volume. Alternative non-local theories have been also resorted to. McFarland and Colton (2005) have built a non-local EB beam based on a continuum micropolar elasticity constitutive law, to be interpreted as the result of long-range forces exerted by non-nearest neighbor atoms connected in the underlying material microstructure of the continuum (Lakes, 1991). Kong et al. (2008) and Zhang et al. (2010) have derived the motion equations of a non-local EB beam by the Hamilton's principle, the first by a modified couple stress theory and the second by a so- 
called hybrid approach, based on a strain energy functional depending on local and non-local curvatures. Wang et al. (2010) have built a non-local TM beam also based on the Hamilton's principle, but in conjunction with the strain gradient elasticity theory proposed by Lam et al. (2003).

Although in the majority of the non-local beam theories developed to date non-local terms are stiffness terms, yet a very interesting and challenging task is a generalization to non-local damping. The subject is of particular relevance since the pioneering work of Russell (1992), who has proposed a non-local damping model for fiber-reinforced composites (such as fiberglass, boron and graphite composites) to account for the dissipation that may be encountered at the fiber-matrix interface, due to imperfect bonding. The physical motivations given by Russell (1992) to pursue a non-local modeling can be briefly summarized as follows. He has considered the specific case of EB composite beams with longitudinal fibers. Starting from the observation that, as the beam undergoes flexural deformation, the movement of the fibers relative to the matrix can be thought as determined by the differential rotation along the beam axis, he has pointed out that the consequent dissipation forces transmitted from the fibers to the matrix determine, within any of the individual beam segments which the fibers pass through, a damping moment. In fact opposite net motions of the fibers relative to the matrix are encountered above and below the elastic axis, when a differential rotation takes place along the beam axis (Russell, 1992). The damping moment acting on a beam segment of unit length has been then built by Russell (1992) as a viscous long-range moment, given in an integral form depending on the relative rate of rotation between the beam segment and the non-adjacent beam segments, through an appropriate attenuation function. In this mathematical form, the dependence on the relative rate of rotation through an attenuation function reflects that, due to presence of the fibers, the motion of the beam segments is inherently coupled and that, as intuition suggests, such a coupling progressively decays with distance. The viscous form, as customary in structural engineering applications, has been intended as a general form accounting for different sources of damping at the fibermatrix interface (friction, plastic deformation of the matrix, etc...). It has to be noted that a viscous long-range moment is involved also in the mechanical boundary conditions (B.C.). Experimental evidence for his non-local damping model has been found by Russell (1992) in the free vibrations of a boron-epoxy composite beam. A similar damping mechanism has been also envisaged by Russell (1992) for the longitudinal damped vibrations of a bar.

Further investigations on non-local damping have been pursued by Banks and coworkers (Banks and Inman, 1991; Banks et al., 1994). For a EB cantilever composite beam with a tip mass, they have found that the internal damping model proposed by Russell (1992), used in conjunction with an external air viscous damping, provide the best fitting to some experimental data (Banks and Inman, 1991). An attempt has been also made to introduce the damping model by Russell (1992) in a TM beam, on the assumption that non-local damping effects do result in an additional damping moment but not in additional damping terms, such as for instance transverse (shear) forces, that may affect the beam equilibrium. In this case, however, results have proved less accurate versus experimental data available (Banks et al., 1994).

More recently, Friswell and coworkers (Lei et al., 2006a,b; Friswell et al., 2007) have proposed a non-local EB beam where non-local damping terms are built as a weighted average of a velocity field over the beam domain, and appropriate attenuation functions are taken as weighting functions. Specifically, external and internal non-local damping models have been considered, the first taken as depending on the transverse displacement, and the second as depending on the fourth-order derivative of the transverse displacement (Sorrentino et al., 2003). While the external damping model is seen as the result of external damping patches, long adhesive joints in composites or surface damping treatments using fluids, Friswell et al. (2007) have pointed out that the internal non-local damping model can be thought of as a "homogenized" model that accounts, in an average sense, for an intrinsic dependence between the response at a given point and the response at the surrounding points of the medium (Flugge, 1975). For instance, a result of such a dependence may be the effects produced at a given point by the complex deformations of non-adjacent beam cross sections, that are not captured in a classical beam model where the cross sections remain planes (Lei et al., 2006a). In this sense, it can be stated that the sources of non-local damping shall be seen, according to Friswell and coworkers, in a more general context than the one envisaged by Russell (1992). Further, considering that the spatial location of the damping sources is generally uncertain, that the damping mechanism itself and its dependence on the material microstructure are difficult to characterize, it may be expected that an internal "homogenized" non-local damping model proves more flexible versus experimental evidence rather than a typical local one (Friswell et al., 2007). In their work, Friswell and coworkers have considered either viscous or viscoelastic non-local damping, the latter with time-dependent exponential forms (Friswell et al., 2007). The resulting motion equation is an integro-differential equation and the free vibration problem has been solved in a discrete form based on the Galerkin method (Lei et al., 2006a,b) and on the finite element method (Friswell et al., 2007).

It has to be noted that the non-local beam model developed by Friswell and coworkers may also include an additional non-local stiffness (Lei et al., 2006a), built on the same mathematical basis as the non-local damping. This is, in the authors' opinion, a relevant feature. Indeed a theoretical model where non-local stiffness and non-local damping are built on the same mathematical basis, and may be considered either separately or simultaneously depending on the material at hand, may be of particular interest in view of the increasing insight that experimental evidence will give into the sources of non-local effects and, also, in view of the new and complex materials that will be produced in the future. In this perspective and in an attempt to contribute to the research effort in this field, a non-local TM beam will be presented in this paper, where non-local stiffness and damping may be accounted for. Specifically, internal damping will be considered.

The mathematical approach shares, with most common nonlocal beam theories, the idea of formulating an enriched continuum model of the beam, where non-local effects are accounted for, in an average sense, by introducing non-local terms in the motion equations. Specifically, the non-local terms are modeled as longrange volume forces and moments, mutually exerted by nonadjacent beam segments, that contribute to the equilibrium of any beam segment, along with the local stress resultants exerted by the adjacent beam segments. Elastic and viscous long-range volume forces/moments can be considered. They are built as linearly depending on the product of the volumes of the interacting beam segments, and on generalized measures of their relative motion, through pertinent attenuation functions governing the space decay of the non-local effects. The generalized measures of relative motion are based on the pure deformation modes of the TM beam derived by Fuchs $(1991,1997)$, i.e. a "pure axial" symmetric mode, a "pure bending" symmetric mode and a "pure shear" asymmetric mode.

In the proposed model, elastic and viscous long-range volume forces/moments can be considered either separately or simultaneously. In this manner, various sources of non-local effects can be addressed, and pertinent applications will be discussed. 
The paper develops in the following steps. The dynamic equilibrium equations of the proposed non-local TM beam are derived in Section 2, along with the mechanical B.C. The elastic and viscous long-range volume forces/moments are introduced in Section 3. The motion equations are derived in Section 4, and numerical results are presented in Section 5. In the Appendix A the proposed non-local TM beam is compared with the previous non-local TM beam built by Di Paola et al. (2011a, 2012).

\section{Formulation}

Consider the beam of arbitrary cross section shown in Fig. 1. The beam is referred to a Cartesian (orthogonal) coordinate system Oxyz where axis $x$ coincides with the centroidal axis, axes $y$ and $z$ are principal axes of the cross section, and $x z$ is the bending plane. Be $\mathbf{x}=\left[\begin{array}{lll}x & y & z\end{array}\right]^{\mathrm{T}}$ the position vector and $L$ the length of the beam. For simplicity, a uniform cross section is considered. The material is isotropic and linearly elastic.

Be $\mathbf{u}(\mathbf{x}, t)$ the displacement vector, $\mathbf{u}^{\mathrm{T}}(\mathbf{x}, t)=\left[\begin{array}{lll}u_{x} & u_{y} & u_{z}\end{array}\right]$. According to the TM beam theory, the small displacement components of a given point $P(x, y, z)$ in the beam can be cast in the form

$u_{x}(\mathbf{x}, t)=u(x, t)-z \varphi(x, t) ; \quad u_{z}(\mathbf{x}, t)=v(x, t) ; \quad u_{y}(\mathbf{x}, t)=0$

where, for a cross section at $x$ on the centroidal axis, $u(x, t), v(x, t)$ and $\varphi(x, t)$ denote the $x$-, $z$-displacement and the rotation about the $y$ axis. The latter is taken as positive if clockwise. The corresponding strain components, as given by the small strain equations, are

$$
\begin{aligned}
\varepsilon(x, t) & =\frac{\partial u(x, t)}{\partial x} ; \quad \gamma(x, t)=\frac{\partial v(x, t)}{\partial x}-\varphi(x, t) \\
\chi(x, t) & =-\frac{\partial \varphi(x, t)}{\partial x}
\end{aligned}
$$

They denote the axial, bending and transverse shear strain, respectively. Further, be

$u\left(x_{i}, t\right)=u_{i} ; v\left(x_{i}, t\right)=v_{i} ; \varphi\left(x_{i}, t\right)=\varphi_{i} ; \quad i=0, L \quad(3 \mathrm{a}-\mathrm{c})$

being $u_{i}, v_{i}$ and $\varphi_{i}$ the displacements at the beam ends, i.e. at $x_{0}=0$ and $x_{L}=L$. That is, time independent B.C. are considered.

Then, be $\boldsymbol{\sigma}^{(l)}(\mathbf{x}, t)=\left[\begin{array}{llllll}\sigma_{x}^{(l)} & \sigma_{y}^{(l)} & \sigma_{z}^{(l)} & \tau_{y z}^{(l)} & \tau_{x z}^{(l)} & \tau_{x y}^{(l)}\end{array}\right]^{\mathrm{T}}$ the vector of the six components of the Cauchy stress tensor and be $N^{(l)}(x, t)$, $T^{(l)}(x, t)$ and $M^{(l)}(x, t)$ the classical local stress resultants, i.e. normal stress, shear stress and bending moment given by

$$
\begin{aligned}
& N^{(l)}(x, t)=\int_{A} \sigma_{x}^{(l)}(\mathbf{X}, t) \mathrm{d} A ; T^{(l)}(x, t)=\int_{A} \tau_{x z}^{(l)}(\mathbf{X}, t) \mathrm{d} A ; \\
& M^{(l)}(x, t)=\int_{A} \sigma_{x}^{(l)}(\mathbf{X}, t) z \mathrm{~d} A .
\end{aligned}
$$

They are related to the corresponding axial, shear and bending strain by the constitutive laws of the TM beam:

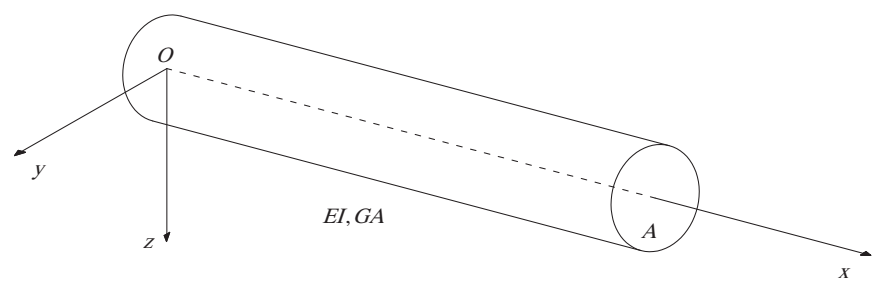

Fig. 1. Timoshenko beam of arbitrary cross section.
$N^{(l)}(x, t)=E^{*} A \varepsilon(x, t)$

$T^{(l)}(x, t)=K_{S} G^{*} A \gamma(x, t) ;$

$M^{(l)}(x, t)=E^{*} I \chi(x, t)$.

In Eq.(5), $A, I$ and $K_{s}$ are the area, the moment of inertia and the TM shear coefficient of the cross section; $E^{*}=\beta_{1} E$ and $G^{*}=\beta_{1} G$, being $E$ and $G$ the Young and the shear modulus; $\beta_{1}$ is a dimensionless coefficient, $0 \leq \beta_{1} \leq 1$, that weighs the amount of local effects (Di Paola et al., 2010b, 2011a). In this respect, note that $\beta_{1}$ is introduced here as in those non-local theories where the non-local material is conceived as a two-phase elastic material (Altan, 1989; Polizzotto, 2001).

Next, divide the beam domain in $N$ segments of length $\Delta x$ and be $x_{i}=i \Delta x$ for $i=0,1, \ldots N-1\left(x_{N}=x_{L}\right)$, the abscissas that define the corresponding positions along the axis. The first, fundamental assumption of the proposed non-local beam is that non-local effects can be modeled as long-range volume forces and moments, mutually exerted by non-adjacent beam segments. This means that the equilibrium of each beam segment is attained due: (i) to the local stress resultants (4) exerted by the adjacent beam segments; (ii) to the resultants of the volume forces/moments exerted by all the non-adjacent beam segments. Then, according to the d'Alembert's principle (Humar, 2002) the dynamic equilibrium equations of the beam segment $\Delta V\left(x_{i}\right)=A \Delta x$ at $x=x_{i}$, for $i=0,1, \ldots N-1$, can be written in the form (see Fig. 2):

$N^{(l)}\left(x_{i}+\Delta x\right)-N^{(l)}\left(x_{i}\right)+Q_{x}\left(x_{i}, t\right)+F_{x}\left(x_{i}, t\right) \Delta x-m\left(x_{i}\right) \ddot{u}\left(x_{i}, t\right) \Delta x=0 ;$

$T^{(l)}\left(x_{i}+\Delta x\right)-T^{(l)}\left(x_{i}\right)+Q_{z}\left(x_{i}, t\right)+F_{z}\left(x_{i}, t\right) \Delta x-m\left(x_{i}\right) \ddot{v}\left(x_{i}, t\right) \Delta x=0 ;$

$M^{(l)}\left(x_{i}+\Delta x\right)-M^{(l)}\left(x_{i}\right)-T^{(l)}\left(x_{i}\right) \Delta x-Q_{\varphi}\left(x_{i}, t\right)+I_{\rho}\left(x_{i}\right) \ddot{\varphi}\left(x_{i}, t\right) \Delta x=0$,

where the dots mean differentiation with respect to time, $F_{x}(x, t)$ and $F_{z}(x, t)$ are introduced as generalized measures per unit length of the external forces on the beam, $m(x)=\rho(x) A$ and $I_{\rho}(x)=\int_{A} \rho(x) z^{2} \mathrm{~d} A$, being $\rho(x)$ the mass per unit volume. Further, $Q_{x}, Q_{z}$ and $Q_{\varphi}$ are the resultants of the long-range volume forces/ moments exerted on the beam segment $\Delta V\left(x_{i}\right)$ at $x=x_{i}$ by all the non-adjacent beam segments $\Delta V\left(\xi_{k}\right)$ at $x=\xi_{k}, \xi_{k} \neq x_{i}$. For brevity, $Q_{x}, Q_{z}$ and $Q_{\varphi}$ will be referred to as long-range resultants.

In the non-local beam model, elastic and damping long-range resultants will be considered, either separately or simultaneously. They are denoted as

$Q_{x}\left(x_{i}, t\right)=R_{x}\left(x_{i}, t\right)+D_{x}\left(x_{i}, t\right) ;$

$Q_{z}\left(x_{i}, t\right)=R_{z}\left(x_{i}, t\right)+D_{z}\left(x_{i}, t\right)$;

$Q_{\varphi}\left(x_{i}, t\right)=R_{\varphi}\left(x_{i}, t\right)+D_{\varphi}\left(x_{i}, t\right)$

where " $R$ " and " $D$ " mean elastic and damping part. The analytical form is specified next.

\section{Long-range resultants}

Both the elastic and the damping long-range resultants are built on a mechanical basis. The key ideas are illustrated first for the elastic long-range resultants and then generalized to the damping ones. 
(a)

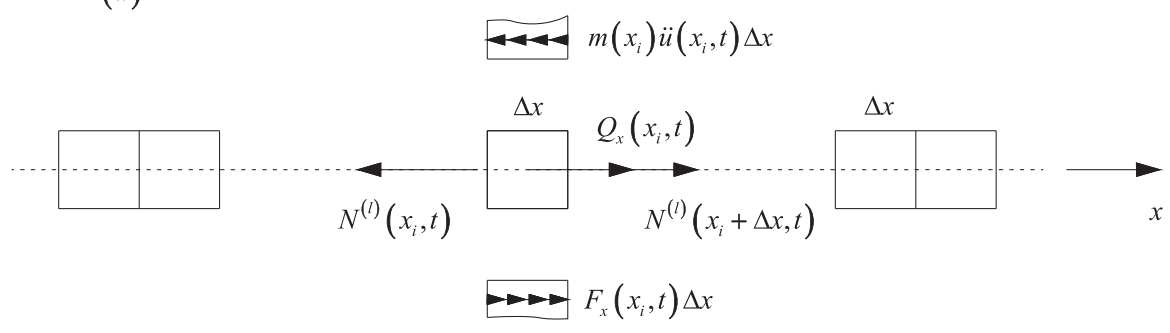

(b)

$$
\text { T4T } m\left(x_{i}\right) \ddot{v}\left(x_{i}, t\right) \Delta x
$$

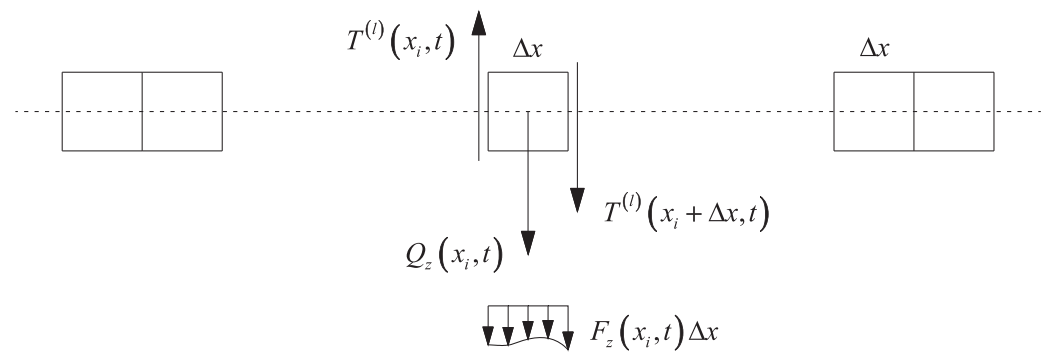

$(c)$

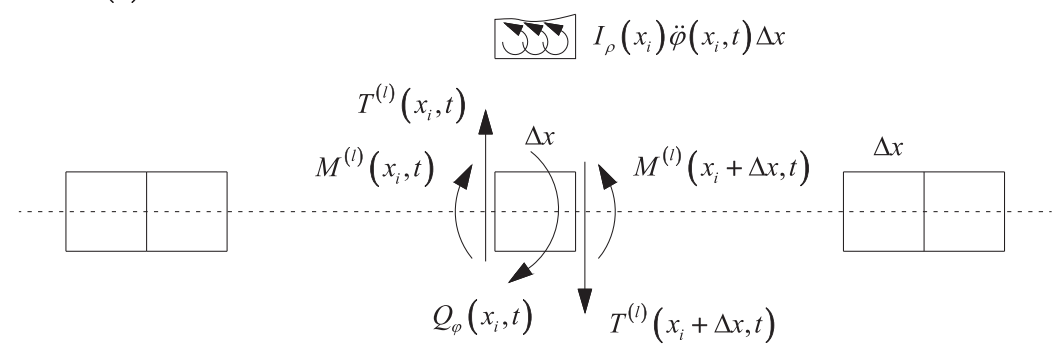

Fig. 2. Equilibrium of an elementary beam segment: (a) axial; (b) transverse; (c) bending. Positive sign conventions are reported.

\subsection{Elastic long-range resultants}

The elastic long-range volume forces/moments mutually exerted by two non-adjacent beam segments $\Delta V\left(x_{i}\right)$ and $\Delta V\left(\xi_{k}\right)$ located, respectively, at $x=x_{i}$ and $x=\xi_{k}$ on the beam axis, are built as a result of a relative motion that may involve, in general, displacements and rotations. Specifically, based on the work by Fuchs $(1991,1997)$ who singled out the pure deformation modes of a TM beam, three separate generalized measures of relative displacements/rotations, that give rise to elastic long-range volume forces/ moments, are used in the non-local beam model. They are conventionally referred to as "pure axial", "pure bending" and "pure shear" deformation mode. The pure axial mode is a symmetric mode, that gives rise to elastic long-range volume axial forces mutually exerted by two non-adjacent beam segments $\Delta V\left(x_{i}\right)$ and $\Delta V\left(\xi_{k}\right)$, as a result of their relative axial displacement

$\eta\left(x_{i}, \xi_{k}, t\right)=u\left(\xi_{k}, t\right)-u\left(x_{i}, t\right)$

The pure bending mode is a symmetric mode, that gives rise to elastic long-range volume moments mutually exerted by two nonadjacent beam segments $\Delta V\left(x_{i}\right)$ and $\Delta V\left(\xi_{k}\right)$, as a result of their relative rotation $\theta\left(x_{i}, \xi_{k}, t\right)=\varphi\left(\xi_{k}, t\right)-\varphi\left(x_{i}, t\right)$

The pure shear mode is an asymmetric mode, that gives rise to elastic long-range volume transverse forces + moments mutually exerted by two non-adjacent beam segments $\Delta V\left(x_{i}\right)$ and $\Delta V\left(\xi_{k}\right)$, as a result of their rotations with respect to the line given by the relative transverse displacement, i.e.

$$
\begin{aligned}
\psi\left(x_{i}, \xi_{k}, t\right)= & {\left[\frac{v\left(\xi_{k}, t\right)-v\left(x_{i}, t\right)}{\xi_{k}-x_{i}}-\varphi\left(\xi_{k}, t\right)\right] } \\
& +\left[\frac{v\left(\xi_{k}, t\right)-v\left(x_{i}, t\right)}{\xi_{k}-x_{i}}-\varphi\left(x_{i}, t\right)\right]
\end{aligned}
$$

In his work, Fuchs $(1991,1997)$ has also derived the selfequilibrated forces/moments at the ends of the TM beam, that result from the pure deformation modes $(8)-(10)$. In the non-local beam model, the elastic long-range volume forces/moments are built based on the analytical form of the self-equilibrated forces/ moments obtained by Fuchs $(1991,1997)$, and introducing a linear dependence on the product of the volumes of the interacting beam segments, through appropriate attenuation functions governing the space decay of non-local effects. Therefore, the long-range resultant $R_{x}\left(x_{i}, t\right)$ is written as 
$R_{X}\left(x_{i}, t\right)=\sum_{k=0, k \neq i}^{N-1} r_{x}\left(x_{i}, \xi_{k}, t\right)$

where $r_{x}\left(x_{i}, \xi_{k}, t\right)$ is the long-range volume axial force exerted on the beam segment $\Delta V\left(x_{i}\right)$ by the non-adjacent beam segment $\Delta V\left(\xi_{k}\right)$ due to the pure axial deformation (8), given by

$r_{x}\left(x_{i}, \xi_{k}, t\right)=\frac{E^{*} A}{\left|x_{i}-\xi_{k}\right|} g_{x}\left(x_{i}, \xi_{k}\right) \eta\left(x_{i}, \xi_{k}, t\right) \Delta V\left(x_{i}\right) \Delta V\left(\xi_{k}\right)$

The long-range resultant $R_{z}\left(x_{i}, t\right)$ is written as

$R_{z}\left(x_{i}, t\right)=\sum_{k=0, k \neq i}^{N-1} r_{z}\left(x_{i}, \xi_{k}, t\right)$,

where $r_{z}\left(x_{i}, \xi_{k}, t\right)$ is the long-range volume transverse force exerted on the beam segment $\Delta V\left(x_{i}\right)$ by the non-adjacent beam segment $\Delta V\left(\xi_{k}\right)$ due to the pure shear deformation mode (10), given by

$r_{z}\left(x_{i}, \xi_{k}, t\right)=\operatorname{sgn}\left(\xi_{k}-x_{i}\right) \frac{6 \mu E^{*} I}{\left|x_{i}-\xi_{k}\right|^{2}} g_{z}\left(x_{i}, \xi_{k}\right) \psi\left(x_{i}, \xi_{k}, t\right) \Delta V\left(x_{i}\right) \Delta V\left(\xi_{k}\right)$,

where $\mu=1 /(1+12 c)$, for $c=E^{*} I / G^{*} A L^{2}$, is a dimensionless parameter depending on the flexural stiffness $E^{*} I$ and the shear stiffness $G^{*} A$, while $\operatorname{sgn}(\xi-x)=+1$ if $(\xi-x)>0$ and $\operatorname{sgn}(\xi-x)=-1$ if $(\xi-x)<0$ is introduced to ensure consistency of $r_{z}(x, \xi, t)$ with the sign convention of the long-range resultants in Fig. 2. Further, the long-range resultant $R_{\varphi}\left(x_{i}, t\right)$ is written as

$R_{\varphi}\left(x_{i}, t\right)=\sum_{k=0, k \neq i}^{N-1} r_{\varphi \varphi}\left(x_{i}, \xi_{k}, t\right)+r_{\varphi z}\left(x_{i}, \xi_{k}, t\right)$,

where $r_{\varphi \varphi}\left(x_{i}, \xi_{k}, t\right)$ and $r_{\varphi z}\left(x_{i}, \xi_{k}, t\right)$ are the long-range volume moments exerted on the beam segment $\Delta V\left(x_{i}\right)$ by the non-adjacent beam segment $\Delta V\left(\xi_{k}\right)$ due to the pure bending deformation (9) and the pure shear deformation (10), given respectively by

$r_{\varphi \varphi}\left(x_{i}, \xi_{k}, t\right)=\frac{E^{*} I}{\left|x_{i}-\xi_{k}\right|} g_{\varphi}\left(x_{i}, \xi_{k}\right) \theta\left(x_{i}, \xi_{k}, t\right) \Delta V\left(x_{i}\right) \Delta V\left(\xi_{k}\right)$

$r_{\varphi z}\left(x_{i}, \xi_{k}, t\right)=\frac{3 \mu E^{*} I}{\left|x_{i}-\xi_{k}\right|} g_{z}\left(x_{i}, \xi_{k}\right) \psi\left(x_{i}, \xi_{k}, t\right) \Delta V\left(x_{i}\right) \Delta V\left(\xi_{k}\right)$

In Eq. (12), Eq. (14) and Eqs. (16) and (17), $g_{x}(x, \xi), g_{z}(x, \xi)$ and $g_{\varphi}(x, \xi)$ are attenuation functions governing the space decay of the nonlocal axial, bending and shear elastic effects. They are taken as symmetric functions with respect to the arguments $x$ and $\xi$, to ensure that the elastic long-range resultants exchanged by the interacting beam segments are mutual, according to Newton's third law (see Figs. 3 and 4). Further, they are taken as positive functions, to ensure that the elastic long-range resultants are restoring terms, at any distance between the interacting beam segments. For generality, in the proposed model $g_{x}(x, \xi), g_{z}(x, \xi)$ and $g_{\varphi}(x, \xi)$ are introduced as independent functions: for instance, they could be given the same mathematical form but with different parameters. This seems an appropriate option in recognition of the fact that nonlocal effects may vary significantly depending on the material microstructure and that, consequently, non-local axial, bending and shear effects shall not necessarily exhibit the same decay. Typical mathematical forms of attenuation functions are exponential, Gaussian or bell-shaped functions (Di Paola et al., 2009;

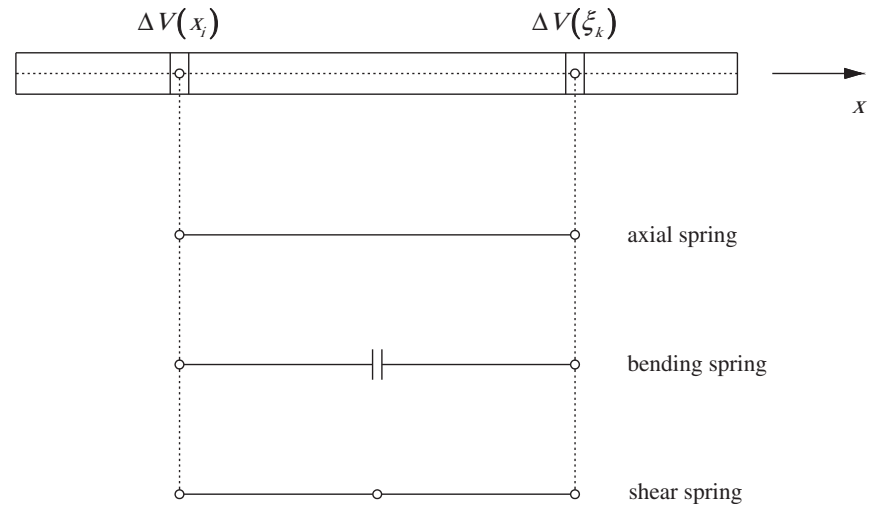

Fig. 3. Mechanics of the long-range interactions between elementary beam segments.

Failla et al., 2013; Friswell et al., 2007). In general, a definite choice on the mathematical form along with the pertinent parameters will depend on the material and shall be obviously based on experimental evidence, through an optimization procedure.

At this stage, a few comments are worth doing. From a mechanical point of view, the elastic long-range volume forces/moments can be interpreted as the result of a spring-like connection between non-adjacent beam segments, where long-range pure axial, pure bending and pure shear springs are accounted for, see Figs. 3 and 4. In this sense, it can be stated that the non-local beam is built on the same mechanical basis as the non-local bar previously developed by the authors (Di Paola et al., 2009, 2011b; Failla et al., 2010), where long-range volume axial forces have been also built as linearly depending on the relative displacement, and on the product of the volumes of the interacting bar segments, through an attenuation function $g(x, \xi)$. It is noted indeed that the long-range resultant (11) can be readily reverted to the long-range resultant involved in the equilibrium of the non-local bar (Di Paola et al., 2009, 2011b; Failla et al., 2010), as long as $g_{x}(x, \xi)=|x-\xi| g(x, \xi) / E^{*} A$ is set.

In addition, it shall be noted that the non-local beam model is a displacement-based model, where the long-range volume forces/ moments arise from relative displacements/rotations between non-adjacent beam segments, that correspond to the pure deformation modes (8)-(10). In this respect, recognize that if the longrange volume transverse forces/moments were taken as depending on the relative transverse displacement and not on the pure shear deformation (10), long-range volume transverse forces/moments would erroneously arise from a relative transverse displacement induced, for instance, by a rigid rotation of the beam. In this sense, it can be stated that the non-local beam model is invariant with respect to rigid body motion and that the axial, bending and shear non-local behaviors are mechanically consistent.

\subsection{Damping long-range resultants}

Although, at least in principle, different sources of non-local damping may be endowed in the non-local beam model, here viscous long-range volume forces/moments are considered. The analytical form is built consistently with the analytical form of the elastic counterparts. That is, it is assumed that two beam segments $\Delta V\left(x_{i}\right)$ and $\Delta V\left(\xi_{k}\right)$, located at $x=x_{i}$ and $x=\xi_{k}$ on the beam axis, may mutually exert viscous long-range volume forces/moments, as a result of the rate of the relative displacements/rotations described in terms of the generalized measures (8)-(10). Still in analogy with the elastic counterparts, the viscous long-range volume forces/ moments are taken as linearly depending on the rate of the 
(a)

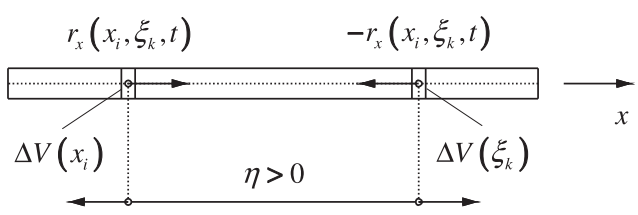

(b)

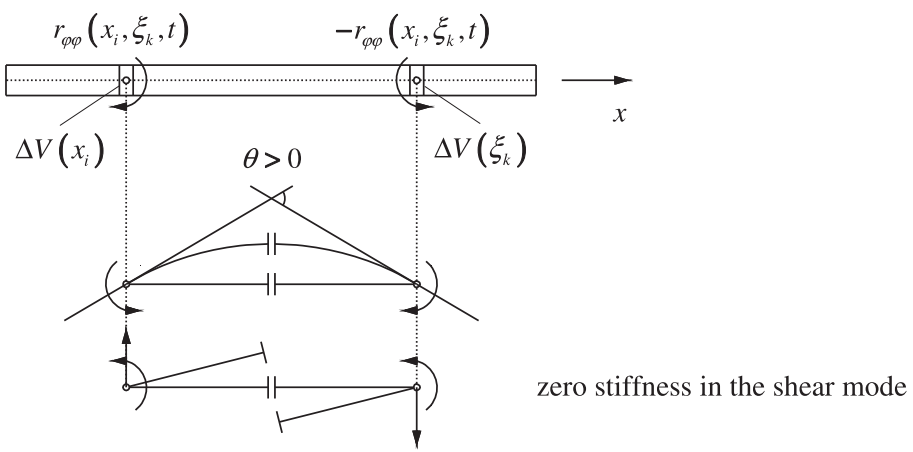

(c)

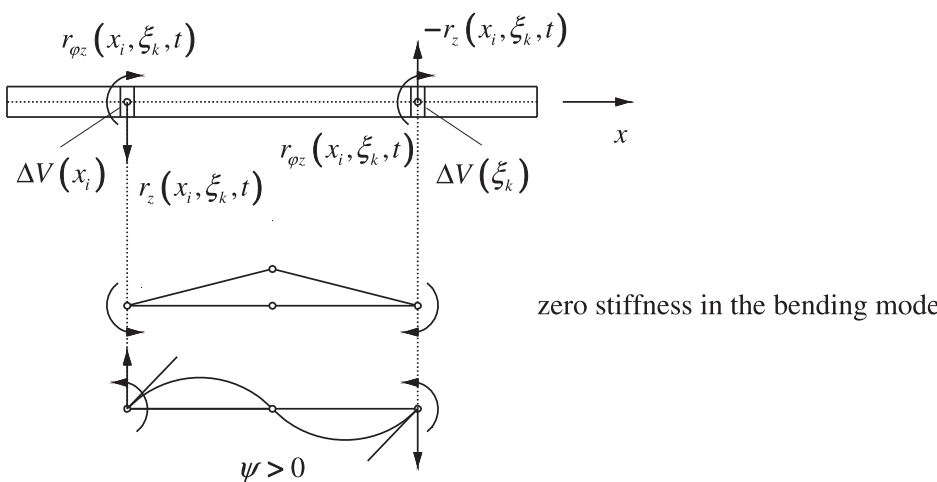

Fig. 4. Long-range springs: (a) axial; (b) pure bending; (c) pure shear.

generalized measures (8)-(10), and on the product of the volumes of the interacting beam segments, through attenuation functions that are pertinent to the non-local viscous damping effects.

Therefore, if $d_{x}(x, \xi, t), d_{z}(x, \xi, t), d_{\varphi \varphi}(x, \xi, t)$ and $d_{\varphi z}(x, \xi, t)$ denote the viscous long-range volume forces/moments exerted on the beam segment $\Delta V\left(x_{i}\right)$ at $x=x_{i}$, by a non-adjacent beam segment of volume $\Delta V\left(\xi_{k}\right)$ at $x=\xi_{k}$, the viscous long-range resultants can be written in the form:

$$
\begin{aligned}
D_{x}\left(x_{i}, t\right) & =\sum_{k=0, k \neq i}^{N-1} d_{x}\left(x_{i}, \xi_{k}, t\right) \\
& =\sum_{k=0, k \neq i}^{N-1} \frac{E^{*} A}{\left|x_{i}-\xi_{k}\right|} \tilde{g}_{x}\left(x_{i}, \xi_{k}\right) \dot{\eta}\left(x_{i}, \xi_{k}, t\right) \Delta V\left(x_{i}\right) \Delta V\left(\xi_{k}\right) ;
\end{aligned}
$$

$$
\begin{aligned}
D_{\varphi}\left(x_{i}, t\right)= & \sum_{k=0, k \neq i}^{N-1} d_{\varphi \varphi}\left(x_{i}, \xi_{k}, t\right)+d_{\varphi z}\left(x_{i}, \xi_{k}, t\right) \\
= & \sum_{k=0, k \neq i}^{N-1} \frac{E^{*} I}{\left|x_{i}-\xi_{k}\right|} \tilde{g}_{\varphi}\left(x_{i}, \xi_{k}\right) \dot{\theta}\left(x_{i}, \xi_{k}, t\right) \Delta V\left(x_{i}\right) \Delta V\left(\xi_{k}\right) \\
& +\sum_{k=0, k \neq i}^{N-1} \frac{3 \mu E^{*} I}{\left|x_{i}-\xi_{k}\right|} \tilde{g}_{z}\left(x_{i}, \xi_{k}\right) \dot{\psi}\left(x_{i}, \xi_{k}, t\right) \Delta V\left(x_{i}\right) \Delta V\left(\xi_{k}\right) .
\end{aligned}
$$

where the dot means differentiation with respect to time. Further, in Eqs. (18)-(20) $\tilde{g}_{x}(x, \xi), \tilde{g}_{z}(x, \xi)$ and $\tilde{g}_{\varphi}(x, \xi)$ are attenuation functions governing the spatial decay of the non-local axial, bending and shear viscous effects. Like the attenuation functions governing the nonlocal elastic effects, they are taken as symmetric functions with

$D_{z}\left(x_{i}, t\right)=\sum_{k=0, k \neq i}^{N-1} d_{z}\left(x_{i}, \xi_{k}, t\right)=\sum_{k=0, k \neq i}^{N-1} \operatorname{sgn}\left(\xi_{k}-x_{i}\right) \frac{6 \mu E^{*} I}{\left|x_{i}-\xi_{k}\right|^{2}} \tilde{g}_{z}\left(x_{i}, \xi_{k}\right) \dot{\psi}\left(x_{i}, \xi_{k}, t\right) \Delta V\left(x_{i}\right) \Delta V\left(\xi_{k}\right) ;$ 
respect to the arguments $x$ and $\xi$, to ensure that the viscous longrange resultants exchanged by interacting beam segments are mutual, according to Newton's third law (see Fig. 4). Also, they are taken as positive functions, to ensure that the viscous long-range resultants are dissipating terms at any distance between the interacting beam segments. For generality, $\tilde{g}_{x}(x, \xi), \tilde{g}_{z}(x, \xi)$ and $\tilde{g}_{\varphi}(x, \xi)$ are introduced as independent functions. As already pointed out in Section 3.1, this option is allowed in recognition of the fact that, depending on the material at hand, non-local axial, bending and shear effects shall not necessarily exhibit the same decay. Typical choices for non-local damping attenuation functions are similar to those for non-local elastic attenuation functions. For instance, see the work by Friswell et al. (2007) where exponential and Gaussian functions have been used for non-local damping effects. A definite choice on the mathematical form along with the pertinent parameters will depend on the material and shall be made based on experimental evidence, through an optimization procedure.

Next, a few final comments on the viscous long-range volume forces/moments are of interest. From a mechanical point of view, they can be interpreted as the result of a dashpot-like connection between non-adjacent beam segments, where pure axial, pure bending and pure shear dashpots are accounted for. The corresponding mechanical model is then analogous to the spring model in Figs. 3 and 4, where springs are replaced by dashpots. It is also apparent that, if the non-local stiffness terms (11), (13), (15) and the non-local damping terms (18)-(20) are considered simultaneously in the model, the long-range volume forces/moments (7) can be interpreted as the result of a non-local viscoelastic connection of the Kelvin-Voigt type.

Finally, it shall be pointed out that the proposed non-local damping model is not proportional, as the viscous long-range volume forces/moments do not exhibit the same analytical form of the (elastic) restoring forces/moments, to which contribute both local and non-local terms.

\section{Dynamics of the non-local beam}

\subsection{Motion equations}

Upon introducing the long-range resultants in Sections 3.1 and 3.2 , it can be readily seen that dividing Eq. (6) by $\Delta x$ and taking the limit $\Delta x \rightarrow 0$ lead to the following equations:

$$
\begin{aligned}
& E^{*} A \frac{\partial^{2} u(x, t)}{\partial x^{2}}+F_{x}(x, t)+A^{2} \int_{0}^{L} \frac{E^{*} A}{|x-\xi|}\left[g_{x}(x, \xi) \eta(x, \xi, t)\right. \\
& \left.\quad+\tilde{g}_{x}(x, \xi) \dot{\eta}(x, \xi, t)\right] \mathrm{d} \xi=m(x) \ddot{u}(x, t) ; \\
& K_{s} G^{*} A\left[\frac{\partial^{2} v(x, t)}{\partial x^{2}}-\frac{\partial \varphi(x, t)}{\partial x}\right]+F_{z}(x, t)+A^{2} \int_{0}^{L} \operatorname{sgn}(\xi-x) \frac{6 \mu E^{*} I}{|x-\xi|^{2}} \\
& \quad \times\left[g_{z}(x, \xi) \psi(x, \xi, t)+\tilde{g}_{z}(x, \xi) \dot{\psi}(x, \xi, t)\right] \mathrm{d} \xi=m(x) \ddot{v}(x, t) ;
\end{aligned}
$$

$$
\begin{aligned}
& E^{*} I \frac{\partial^{2} \varphi(x, t)}{\partial x^{2}}+K_{s} G^{*} A\left[\frac{\partial v(x, t)}{\partial x}-\varphi(x, t)\right]+A^{2} \int_{0}^{L} \frac{E^{*} I}{|x-\xi|} \\
& \quad \times\left[g_{\varphi}(x, \xi) \theta(x, \xi, t)+\tilde{g}_{\varphi}(x, \xi) \dot{\theta}(x, \xi, t)\right] \mathrm{d} \xi+A^{2} \int_{0}^{L} \frac{3 \mu E^{*} I}{|x-\xi|} \\
& \quad \times\left[g_{z}(x, \xi) \psi(x, \xi, t)+\tilde{g}_{z}(x, \xi) \dot{\psi}(x, \xi, t)\right] \mathrm{d} \xi=I_{\rho}(x) \ddot{\varphi}(x, t),
\end{aligned}
$$

where the constitutive local laws (5) have been introduced and $\Delta V(x)=A \Delta x, \Delta V(\xi)=A \Delta \xi$ have been set for the volumes of the interacting beam segments. As concerns the B.C. it can be seen that, in the dynamic equilibrium equations at the beam ends, the longrange resultants (11), (13), (15), (18)-(20) can be considered as infinitesimal of higher order with respect to the local stress resultants (see Di Paola et al., 2009). For this reason, the mechanical B.C. hold in the classical form of local theory:

$$
\begin{aligned}
& \left.E^{*} A \frac{\partial u(x, t)}{\partial x}\right|_{x=x_{i}}=\mp N_{i}(t) \\
& \left.K_{S} G^{*} A\left[\frac{\partial v(x, t)}{\partial x}-\varphi(x, t)\right]\right|_{x=x_{i}}=\mp T_{i}(t) ; \\
& \left.E^{*} I \frac{\partial \varphi(x, t)}{\partial x}\right|_{x=x_{i}}=\mp M_{i}(t) ;
\end{aligned}
$$

where $N_{i}, M_{i}$ and $T_{i}$ are the external forces/moments acting at the ends of the beam $(i=0, L)$.

Eqs. (21)-(23) are the motion equations of the proposed nonlocal TM beam model. It is a continuum model, where the nonlocal effects are accounted for, in an average sense, by the integral terms in the 1.h.s. of Eqs. (21)-(23). They represent the elastic and viscous long-range resultants per unit length. The following few remarks are now worth doing on the applications.

Remark 1. If elastic long-range resultants only are considered in Eqs. (21)-(23), the proposed model is a TM beam model with nonlocal elastic effects. It is suitable for typical applications of alternative existing non-local beam theories, as for instance to capture small-size effects in micro- or nano-beams. An example will be given in Section 5.1. It shall be also pointed out that in a non-local TM beam previously introduced by Di Paola et al. (2011a, 2012) within the more general formulation of a non-local 3D continuum (Di Paola et al., 2010a,b), elastic long-range resultants per unit length are also involved in the equilibrium equations. The relation between the non-local beam by Di Paola et al. (2011a, 2012) and the non-local beam of this paper will be discussed in the Appendix A.

Remark 2. If viscous long-range resultants only are considered in Eqs. (21) -(23), the proposed model is a TM beam model with nonlocal viscous effects. In this case, it can be seen as a generalization of the model by Russell (1992) to address, for instance, non-local damping effects in fiber-reinforced composite beams. It can be noted indeed that the viscous long-range axial force per unit length in Eq. (21) and the viscous long-range moment per unit length in Eq. (23), specifically the part due to the pure bending mode (9), have respectively the same mathematical form of the viscous longrange axial force and the viscous moment per unit length introduced by Russell (1992): they account for the dissipation forces transmitted to the matrix from fibers as a result of a differential axial displacement and a differential rotation along the beam axis, in a beam with longitudinal fibers.

With respect to the model by Russell (1992), however, the proposed model introduces some important novelties. The motion equations (21)-(23) involve, along with the viscous long-range terms already considered by Russell (1992), the viscous longrange terms due to the pure shear deformation mode (10). The presence of these terms allows the proposed model to be applied to beams where embedded fibers may be also inclined with respect to the beam axis. In fact in such beams, along with the dissipation forces due to a differential axial displacement and a differential rotation along the beam axis, it shall be also accounted for the dissipation forces transmitted to the matrix from the inclined fibers, as a result of a shear deformation along the beam axis. Based 
on the same reasoning by Russell (1992), it can be readily seen that these dissipation forces will generally result, within any beam segment which the inclined fibers pass through, in an additional transverse force and an additional moment: on a beam segment of unit length, they are indeed the viscous long-range transverse force in Eq. (22) and the viscous long-range moment in Eq. (23) of the proposed model, due to the pure shear deformation mode (10).

In light of the considerations above it can be concluded that, when viscous long-range resultants only are considered in Eqs. (21)-(23), the proposed model includes and generalizes the previous model by Russell (1992). Specifically, when viscous longrange moments due to the pure bending mode (9) and longrange transverse forces/moments due to the pure shear deformation mode (10) are considered in Eqs. (22) and (23), the proposed model can be applied to study the flexural response of composite beams with multi-oriented 2D fiber reinforcements: a typical example is the in-plane flexural response of symmetric or quasiisotropic laminates (Campbell, 2010). On the other hand, when viscous long-range moments due to the pure bending mode (9) only are considered in Eq. (23), the proposed model can be applied, as the model by Russell (1992), to study the flexural response of composite beams with longitudinal fibers: typical example is the out-of-plane flexural response of unidirectional laminates.

As a further comment on the generalizations introduced by the proposed model, it shall be also pointed out that the proposed model applies to shear-deformable TM beams, while the model by Russell (1992) applies to EB beams only. Finally, it is noted that in the proposed model the mechanical B.C. do not involve non-local terms, and are identical to those of the classical local theory.

Remark 3. If elastic and viscous long-range resultants are considered simultaneously in Eqs. (21)-(23), the proposed model is a TM beam model with non-local Kelvin-Voigt viscoelastic effects. It can be readily seen, in fact, that the total long-range resultants in Eqs. (21)-(23) are viscoelastic terms of the classical Kelvin-Voigt type. In this case, the proposed model can be seen as a further development of the model by Russell (1992) for fiber-reinforced composite beams, in the sense that the long-range resultants in Eqs. (21)-(23) now account for a different dissipation mechanism at the fiber-matrix interface, i.e. viscoelastic and not purely viscous as in the model by Russell (1992). Several studies have shown that, in some fiber-reinforced composites, a viscoelastic modeling of the dissipation mechanism at the fiber-matrix interface has to be pursued (Gosz et al., 1991). In general, it may account for mechanical imperfections, unreacted polymer components, fiber treatments or, in some cases, for the presence of an "engineered" interphase between fibers and matrix, to optimize composite performances (Matzenmiller and Gerlach, 2004; Fisher and Brinson, 2001).

Based on the considerations made in Remark 2 it can be stated that, as in the case of viscous long-range resultants, also in this case the proposed model applies to beams with longitudinal or multioriented 2D fiber reinforcements. An example will be given in Section 5.2. To substantiate the interest that is currently devoted to non-local viscoelastic models for fiber-reinforced composites, it is also worth noting that an alternative theoretical non-local viscoelastic model has been recently proposed by Zhao et al. (2008) for a bar in tension, based on a generalized strain gradient viscoelasticity theory.

\subsection{Free vibration problem}

Attention will be now devoted to the solution of the free vibration bending problem, governed by Eqs. (22) and (23) for
$F_{z}(x, t)=0$. Here, the solution is found based on a finite difference discretization of the integro-differential equations (22) and (23). Upon discretizing the beam domain in $N$ segments $\Delta x\left(x_{i}=i \Delta x\right.$ for $i=0,1, \ldots, N-1)$, the following forms are then adopted for the differential terms

$$
\begin{aligned}
& \left.K_{S} G^{*} A\left[\frac{\partial^{2} v(x, t)}{\partial x^{2}}-\frac{\partial \varphi(x, t)}{\partial x}\right]\right|_{x=x_{i}} \\
& \approx K_{S} G^{*} A\left[\frac{v\left(x_{i+1}, t\right)+v\left(x_{i-1}, t\right)-2 v\left(x_{i}, t\right)}{\Delta x^{2}}-\frac{\varphi\left(x_{i+1}, t\right)-\varphi\left(x_{i-1}, t\right)}{2 \Delta x}\right]
\end{aligned}
$$

$$
\begin{gathered}
\left.E^{*} I \frac{\partial^{2} \varphi(x, t)}{\partial x^{2}}\right|_{x=x_{i}}+\left.K_{S} G^{*} A\left[\frac{\partial v(x, t)}{\partial x}-\varphi(x, t)\right]\right|_{x=x_{i}} \\
\approx E^{*} I \frac{\varphi\left(x_{i+1}, t\right)+\varphi\left(x_{i-1}, t\right)-2 \varphi\left(x_{i}, t\right)}{\Delta x^{2}} \\
\quad+K_{S} G^{*} A\left[\frac{v\left(x_{i+1}, t\right)-v\left(x_{i-1}, t\right)}{2 \Delta x}-\varphi\left(x_{i}, t\right)\right] .
\end{gathered}
$$

The integral terms are reverted to discrete summations such as, for instance (the corresponding discrete expressions for the integral terms in Eq. (23) are not reported for brevity)

$$
\begin{aligned}
& A^{2} \int_{0}^{L} \operatorname{sgn}(\xi-x) \frac{6 \mu E^{*} I}{|x-\xi|^{2}}\left[g_{z}(x, \xi) \psi(x, \xi, t)+\tilde{g}_{z}(x, \xi) \dot{\psi}(x, \xi, t)\right] \mathrm{d} \xi \\
& \approx A^{2} \sum_{k=0, k \neq i}^{N-1} \operatorname{sgn}\left(\xi_{k}-x_{i}\right) \frac{6 \mu E^{*} I}{\left|x_{i}-\xi_{k}\right|^{2}} \\
& \quad \times\left[g_{z}\left(x_{i}, \xi_{k}\right) \psi\left(x_{i}, \xi_{k}, t\right)+\tilde{g}_{z}\left(x_{i}, \xi_{k}\right) \dot{\psi}\left(x_{i}, \xi_{k}, t\right)\right] \Delta x .
\end{aligned}
$$

Then, assuming the standard form $\mathbf{U}=\Psi e^{s t}$ for the displacement/ rotation response involved in the discretized Eqs. (22) and (23) leads to the following complex eigenvalue problem

$$
\left[s^{2} \mathbf{M}+s \mathbf{C}+\mathbf{K}\right] \mathbf{\Psi}=0
$$

where $\mathbf{M}, \mathbf{K}$ and $\mathbf{C}$ are a mass, a stiffness and damping matrix, $\boldsymbol{\Psi}$ is the eigenvector corresponding to the eigenvalue $s$.

To solve Eq. (28) the Galerkin method is applied. This approach has been successfully adopted by Lei et al. (2006a) for non-local and non-proportional damping. Specifically, the following expression is considered for the sought eigenvector $\boldsymbol{\Psi}$

$\boldsymbol{\Psi}=\sum_{i=1}^{M} c_{i} \boldsymbol{\Phi}_{i}$,

where $\boldsymbol{\Phi}_{i}$ for $i=1,2, \ldots M$, are the eigenvectors of the undamped beam. Upon computing the complex eigenvalues $s_{j}$, the damping ratios $\zeta_{j}$ are derived as $\zeta_{j}=-\Re\left(s_{j}\right) /\left|s_{j}\right|$. Obviously, whereas nonlocal effects are considered only in the stiffness (i.e., $\mathbf{C}=\mathbf{0}$ in Eq. (28)), the non-local natural frequencies and the corresponding eigenvectors can be determined by standard methods of eigenanalysis.

\section{Numerical applications}

The free vibration flexural response of the proposed non-local TM beam model is investigated in two cases. In the first, an epoxy micro-beam with non-local elastic effects only is considered, 
i.e. only elastic long-range resultants are considered in Eqs. (22) and (23). In the second, a 2D isotropic laminated fiberglass/polyester beam with non-local Kelvin-Voigt viscoelastic effects is considered, i.e. elastic and viscous long-range resultants are simultaneously included in Eqs. (22) and (23).

\subsection{Undamped free vibrations of a TM beam with non-local elastic effects}

A simply supported epoxy micro-beam with the following parameters is considered: $L=300 \mu \mathrm{m}$, rectangular cross section of width $b=100 \mu \mathrm{m}$ and variable height $h$, Young's modulus $E=1.40 \mathrm{GPa}, \nu=0.35$, mass density $\rho=1200 \mathrm{~kg} \mathrm{~m}^{-3}$.

It is assumed that the non-local bending and shear behaviors are governed by the same attenuation functions, i.e.

$g_{q}(x, \xi)=g(x, \xi), \quad$ for $q=\varphi, z$;

where $g(x, \xi)$ is given by

$g(x, \xi)=C_{r} \exp \left(-\frac{|x-\xi|}{l_{r}}\right)$.

It is worth noting that the exponential function (31) is commonly used as attenuation function in many non-local theories. For instance, it has been used for a non-local stiffness model by Friswell et al. (2007). It is symmetric with respect to the arguments and positive, i.e. it meets the requirements given in Section 3.1. Obviously, there exist alternative attenuation functions with similar properties, and examples can be found in Eringen (1972, 1983, 1987), Polizzotto (2001), Friswell et al. (2007). Pertinent applications are not included for brevity.

In Eq. (31) $C_{r}$ is a constant, $l_{r}$ is the internal length. The larger is the internal length, the wider is the so-called influence distance, i.e. the maximum distance beyond which the attenuation functions and therefore the non-local effects become negligible. The numerical values $C_{r}$ and $l_{r}$ will be set on a theoretical basis, in order to enhance non-local effects and assess how they affect the free vibration solution. Specifically, $C_{r}=10^{10} \mathrm{~m}^{-6}$ is set in Eq. (31), while different values of the internal length $l_{r}$ are considered. Note that lower values of $C_{r}\left(C_{r}<10^{10} \mathrm{~m}^{-6}\right)$ would not result in appreciable non-local elastic effects with respect to local ones and, for this reason, they have been disregarded.

In the constitutive equations (5) $\beta_{1}=1$ is selected. As a result of this choice, the non-local solution will tend to the solution obtained by the classical local TM theory, as $l_{r} \rightarrow 0$ in $g(x, \xi)$ given by Eq. (31). The numerical results reported are obtained for $N=800$ intervals in the beam domain. No significant differences are encountered for $N>800$. The first four natural frequencies $\omega_{j}^{(n l)}=-\mathrm{i} s_{j}$ (i is the imaginary unit) are given in Table 1 .

Regardless of the $L / h$ ratio, it is seen that the natural frequencies are higher than the corresponding values of the classical local TM theory (i.e., the values corresponding to $l_{r}=0$, for which $g(x, \xi)=0$ in Eq. (31)). This result can be explained considering that the elastic long-range interactions between non-adjacent beam segments provide additional stiffness. It is also noted that the natural frequencies increase with the internal length $l_{r}$. A larger internal length $l_{r}$ corresponds indeed to a larger amount of mutually interacting non-adjacent beam segments, with a consequent stiffening of the solution. Further, as concerns the dependence on the $L / h$ ratio, it is seen that the ratio of the natural frequencies to the corresponding values of the classical local TM theory increases as the $L / h$ ratio decreases, i.e. as the beam height increases ( $L$ is held constant). This result shall be attributed to the fact that higher values of $h$ determine an increased magnitude of the elastic long-
Table 1

Undamped free vibrations of an epoxy micro-beam: natural frequencies (MHz).

\begin{tabular}{cccccc}
\hline & $l_{r}(\mu \mathrm{m})$ & Mode 1 & Mode 2 & Mode 3 & Mode 4 \\
\hline$L / h=20$ & 0.0 & 0.08134 & 0.32125 & 0.70833 & 1.22614 \\
& 0.5 & 0.08166 & 0.32510 & 0.72575 & 1.27647 \\
& 1.0 & 0.08199 & 0.32664 & 0.73002 & 1.28589 \\
& 3.0 & 0.08520 & 0.33959 & 0.75952 & 1.33920 \\
& 6.0 & 0.09517 & 0.37931 & 0.84830 & 1.49565 \\
& 9.0 & 0.10975 & 0.43696 & 0.97592 & 1.71809 \\
& 12.0 & 0.12729 & 0.50553 & 1.12575 & 1.97616 \\
& 15.0 & 0.14661 & 0.57986 & 1.28597 & 2.24892 \\
& & & & & \\
& 0.0 & 0.16059 & 0.61294 & 1.28933 & 2.11919 \\
& 0.5 & 0.16278 & 0.64207 & 1.41275 & 2.43889 \\
& 1.0 & 0.16345 & 0.64526 & 1.42151 & 2.45750 \\
& 3.0 & 0.16986 & 0.67088 & 1.47906 & 2.55922 \\
& 6.0 & 0.18974 & 0.74937 & 1.65195 & 2.85797 \\
& 9.0 & 0.21881 & 0.86327 & 1.90033 & 3.28248 \\
& 12.0 & 0.25378 & 0.99868 & 2.19179 & 3.77456 \\
& 15.0 & 0.29230 & 1.14545 & 2.50325 & 4.29416 \\
& & & & & \\
& 0.0 & 0.30645 & 1.05955 & 2.01861 & 3.05630 \\
& 0.5 & 0.32162 & 1.22696 & 2.57494 & 4.21311 \\
& 1.0 & 0.32295 & 1.23300 & 2.58966 & 4.23949 \\
& 3.0 & 0.33561 & 1.28189 & 2.69357 & 4.41099 \\
& 6.0 & 0.37489 & 1.43173 & 3.00763 & 4.92322 \\
& 9.0 & 0.43230 & 1.64905 & 3.45839 & 5.65009 \\
& 12.0 & 0.50136 & 1.90721 & 3.98637 & 6.49013 \\
& 15.0 & 0.57742 & 2.18680 & 4.54944 & 7.37416 \\
\hline \multirow{5}{*}{$5=$} & & & &
\end{tabular}

range resultants (as in fact the stiffness $E^{*} I$ is involved in the long-range resultants in Eqs. (22) and (23)) and, consequently, a stiffening effect. It is interesting to note that experimental evidence of an increased stiffness with respect to the classical local solutions, due to non-local microstructural effects in micro-beams, has been found (Lam et al., 2003; McFarland and Colton, 2005), and is also encountered in the theoretical models devised by Lam et al. (2003) based on strain gradient elasticity and by McFarland and Colton (2005) based on micropolar elasticity.

Fig. 5 shows the ratio $\omega^{(n l)} / \omega^{(l)}$ of the first four non-local natural frequencies, $\omega^{(n l)}$, to the corresponding local values, $\omega^{(l)}$, for $L / h=5$ and the internal lengths $l_{r}$ considered in Table 1 . The non-local natural frequencies increase with the internal length $l_{r}$, approximately with the same rate. It can be also noted that the ratio $\omega^{(n l)} / \omega^{(l)}$ is higher for the higher vibration modes. This result is due to the fact that the non-local beam model is displacement based: the higher vibration modes involve a larger relative motion between

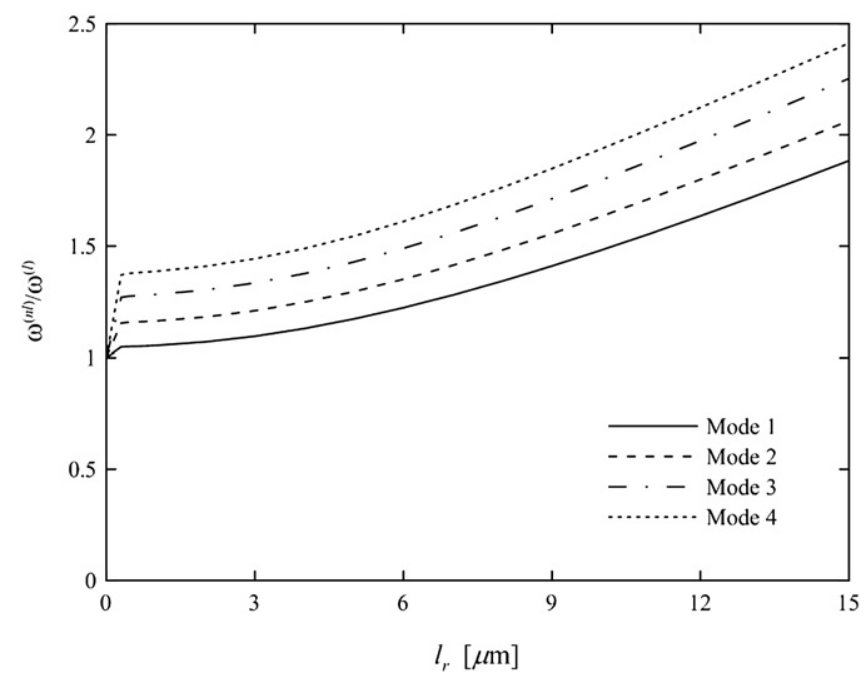

Fig. 5. Ratio of non-local to local natural frequencies of an epoxy micro-beam, for $L /$ $h=5$ and different internal lengths $l_{r}$ 

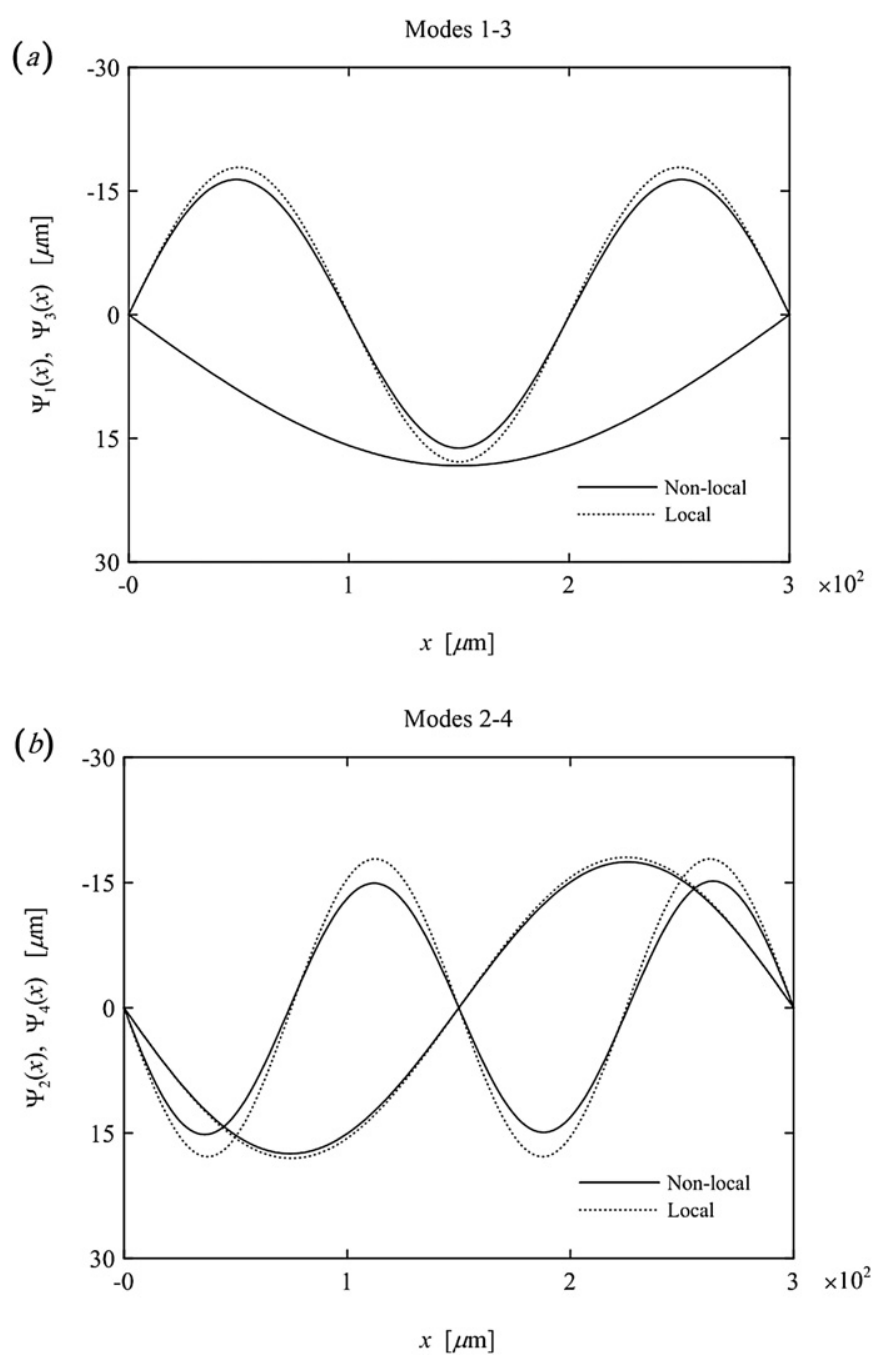

Fig. 6. Non-local modes of an epoxy micro-beam: (a) 1st and 3rd symmetric modes; (b) 2nd and 4th anti-symmetric modes.

non-adjacent beam segments and, as such, they are affected by a larger amount of elastic long-range interactions, with a consequent stiffening of the solution.

Fig. 6 shows the first four vibration modes of the non-local beam, for $L / h=5$ and $l_{r}=15 \mu \mathrm{m}$, along with the corresponding local modes. They are all normalized with respect to the mass matrix and then scaled by a factor $10^{-6}$. The modes are symmetric or anti-symmetric as in the local case. No very significant changes are encountered with respect to the local vibration modes, in agreement with the results provided by different non-local beam theories (Xu, 2006).

\subsection{Damped free vibrations of a TM beam with non-local viscoelastic effects}

The in-plane flexural response of a 2D isotropic laminated beam is considered. The laminate is made of polyester resin with a fiberglass reinforcement. The beam is simply supported. The following geometry/parameters are selected: $L=1 \mathrm{~m}$, rectangular cross section of width $b=5 \mathrm{~mm}$ and variable height $h$, Young's modulus $E=28.0 \mathrm{GPa}, G=4.0 \mathrm{GPa}$, mass density $\rho=1800 \mathrm{~kg} \mathrm{~m}^{-3}$.

It is assumed that pure bending and pure shear behaviors are governed by the same attenuation functions. Among the many potential attenuation functions, an exponential form is considered for both elastic and viscous non-local effects, i.e.

$$
\begin{aligned}
& g_{q}(x, \xi)=g(x, \xi)=C_{r} \exp \left(-\frac{|x-\xi|}{l_{r}}\right), \quad \text { for } q=\varphi, z, \\
& \tilde{g}_{q}(x, \xi)=\tilde{g}(x, \xi)=C_{d} \exp \left(-\frac{|x-\xi|}{l_{d}}\right), \quad \text { for } q=\varphi, z
\end{aligned}
$$

An exponential form analogous to Eqs. (32a) and (32b) has been proposed by Friswell et al. (2007) in his beam model with non-local stiffness and damping. Both are symmetric with respect to the arguments and positive, i.e. they meet the requirements given in Sections 3.1 and 3.2. As already pointed out in Section 5.1, there exist alternative attenuation functions with similar properties, examples can be found in Eringen (1972, 1983, 1987), Polizzotto (2001), Friswell et al. (2007) and pertinent applications are not included for brevity.

In Eq. (32) $C_{r}$ and $C_{d}$ are two constants, $l_{r}$ and $l_{d}$ are internal lengths governing the spatial decay of elastic and viscous non-local effects. Here, the respective numerical values will be set on a theoretical basis, in order to enhance non-local effects and assess how they affect the free vibration solution. They will be thought as independent parameters, i.e. $C_{r} \neq C_{d}$ and $l_{r} \neq l_{d}$. In this manner, it is ensured that the stiffness and viscous damping parameters of the long-range viscoelastic interactions are not identical, as generally encountered in any viscoelastic model. On the other hand, allowing $l_{r} \neq l_{d}$ means that the stiffness and the viscous damping parameters

Table 2

\begin{tabular}{|c|c|c|c|c|c|}
\hline & $l_{d}(\mathrm{~m})$ & Mode 1 & Mode 2 & Mode 3 & Mode 4 \\
\hline$L / h=20$ & $\begin{array}{l}0.02 \\
0.04 \\
0.06 \\
0.08 \\
0.10\end{array}$ & $\begin{aligned}-5.2 & \pm 2350.3 \mathrm{i} \\
-9.7 & \pm 2350.3 \mathrm{i} \\
-16.2 & \pm 2350.3 \mathrm{i} \\
-24.6 & \pm 2350.2 \mathrm{i} \\
-34.5 & \pm 2350.1 \mathrm{i}\end{aligned}$ & $\begin{array}{l}-121.4 \pm 8254.4 \mathrm{i} \\
-197.6 \pm 8255.6 \mathrm{i} \\
-295.2 \pm 8253.7 \mathrm{i} \\
-412.1 \pm 8248.6 \mathrm{i} \\
-544.0 \pm 8240.2 \mathrm{i}\end{array}$ & $\begin{aligned}-588.8 & \pm 16,279.9 \mathrm{i} \\
-947.0 & \pm 16,280.0 \mathrm{i} \\
-1395.0 & \pm 16,249.3 \mathrm{i} \\
-1922.2 & \pm 16,188.7 \mathrm{i} \\
-2505.6 & \pm 16,098.2 \mathrm{i}\end{aligned}$ & $\begin{array}{l}-1699.7 \pm 25,518.6 \mathrm{i} \\
-2751.4 \pm 25,460.7 \mathrm{i} \\
-4063.3 \pm 25,259.5 \mathrm{i} \\
-5575.5 \pm 24,924.9 \mathrm{i} \\
-7196.3 \pm 24,460.1 \mathrm{i}\end{array}$ \\
\hline$L / h=10$ & $\begin{array}{l}0.02 \\
0.04 \\
0.06 \\
0.08 \\
0.10\end{array}$ & $\begin{aligned}-28.8 & \pm 4684.8 \mathrm{i} \\
-47.3 & \pm 4685.4 \mathrm{i} \\
-73.3 & \pm 4685.4 \mathrm{i} \\
-106.6 & \pm 4684.9 \mathrm{i} \\
-146.1 & \pm 4683.9 \mathrm{i}\end{aligned}$ & $\begin{aligned}-664.3 & \pm 16,307.3 \mathrm{i} \\
-958.0 & \pm 16,328.7 \mathrm{i} \\
-1336.4 & \pm 16,308.7 \mathrm{i} \\
-1794.5 & \pm 16,258.4 \mathrm{i} \\
-2312.6 & \pm 16,179.8 \mathrm{i}\end{aligned}$ & $\begin{aligned}-2809.4 & \pm 32,365.3 \mathrm{i} \\
-4069.0 & \pm 32,310.7 \mathrm{i} \\
-5787.5 & \pm 31,953.7 \mathrm{i} \\
-7855.3 & \pm 31,370.1 \mathrm{i} \\
-10,142.7 & \pm 30,562.4 \mathrm{i}\end{aligned}$ & $\begin{aligned}-6118.7 & \pm 50,920.2 \mathrm{i} \\
-9946.8 & \pm 50,032.8 \mathrm{i} \\
-15,130.0 & \pm 48,248.1 \mathrm{i} \\
-21,070.8 & \pm 45,505.5 \mathrm{i} \\
-27,335.3 & \pm 41,631.4 \mathrm{i}\end{aligned}$ \\
\hline$L / h=5$ & $\begin{array}{l}0.02 \\
0.04 \\
0.06 \\
0.08 \\
0.10\end{array}$ & $\begin{array}{l}-203.2 \pm 9277.6 \mathrm{i} \\
-280.2 \pm 9286.3 \mathrm{i} \\
-382.2 \pm 9287.6 \mathrm{i} \\
-512.0 \pm 9283.5 \mathrm{i} \\
-666.2 \pm 9274.0 \mathrm{i}\end{array}$ & $\begin{aligned}-3285.8 & \pm 32,156.7 \mathrm{i} \\
-4188.2 & \pm 32,146.5 \mathrm{i} \\
-5578.7 & \pm 31,819.0 \mathrm{i} \\
-7331.8 & \pm 31,297.5 \mathrm{i} \\
-9321.7 & \pm 30,587.1 \mathrm{i}\end{aligned}$ & $\begin{aligned}-7701.9 & \pm 63,081.4 \mathrm{i} \\
-12,220.3 & \pm 61,551.1 \mathrm{i} \\
-18,857.3 & \pm 58,887.6 \mathrm{i} \\
-26,587.5 & \pm 54,882.1 \mathrm{i} \\
-34,832.3 & \pm 49,159.8 \mathrm{i}\end{aligned}$ & $\begin{aligned}-11,352.1 & \pm 92,062.5 \mathrm{i} \\
-24,322.6 & \pm 87,862.7 \mathrm{i} \\
-41,577.9 & \pm 79,340.0 \mathrm{i} \\
-60,607.8 & \pm 64,015.7 \mathrm{i} \\
-80,006.6 & \pm 34,312.7 \mathrm{i}\end{aligned}$ \\
\hline
\end{tabular}

Damped free vibrations of a fiberglass-polyester composite beam: complex eigenvalues. 
Table 3

Damped free vibrations of a fiberglass-polyester composite beam: damping ratios.

\begin{tabular}{llllll}
\hline & $l_{d}(\mathrm{~m})$ & Mode 1 & Mode 2 & Mode 3 & Mode 4 \\
\hline$L / h=20$ & 0.02 & 0.00223 & 0.01470 & 0.03614 & 0.06645 \\
& 0.04 & 0.00415 & 0.02393 & 0.05807 & 0.10744 \\
& 0.06 & 0.00691 & 0.03574 & 0.08553 & 0.15882 \\
& 0.08 & 0.01046 & 0.04990 & 0.11791 & 0.21829 \\
& 0.10 & 0.01468 & 0.06587 & 0.15379 & 0.28224 \\
$L / h=10$ & 0.02 & 0.00615 & 0.04070 & 0.08647 & 0.11930 \\
& 0.04 & 0.01011 & 0.05857 & 0.12494 & 0.19499 \\
& 0.06 & 0.01565 & 0.08167 & 0.17822 & 0.29922 \\
& 0.08 & 0.02275 & 0.10971 & 0.24290 & 0.42018 \\
& 0.10 & 0.03118 & 0.14149 & 0.31497 & 0.54886 \\
& 0.02 & 0.02189 & 0.10165 & 0.12119 & 0.12238 \\
& 0.04 & 0.03016 & 0.12919 & 0.19473 & 0.26679 \\
& 0.06 & 0.04112 & 0.17269 & 0.30497 & 0.46417 \\
& 0.08 & 0.05507 & 0.22808 & 0.43598 & 0.68751 \\
& 0.10 & 0.07166 & 0.29152 & 0.57813 & 0.91904 \\
\hline
\end{tabular}

may exhibit a different spatial decay. It is believed that, in practical applications, this option may ensure a better fit of the proposed model to experimental evidence, in recognition of the fact that the long-range resultants are not built based on an exact description of
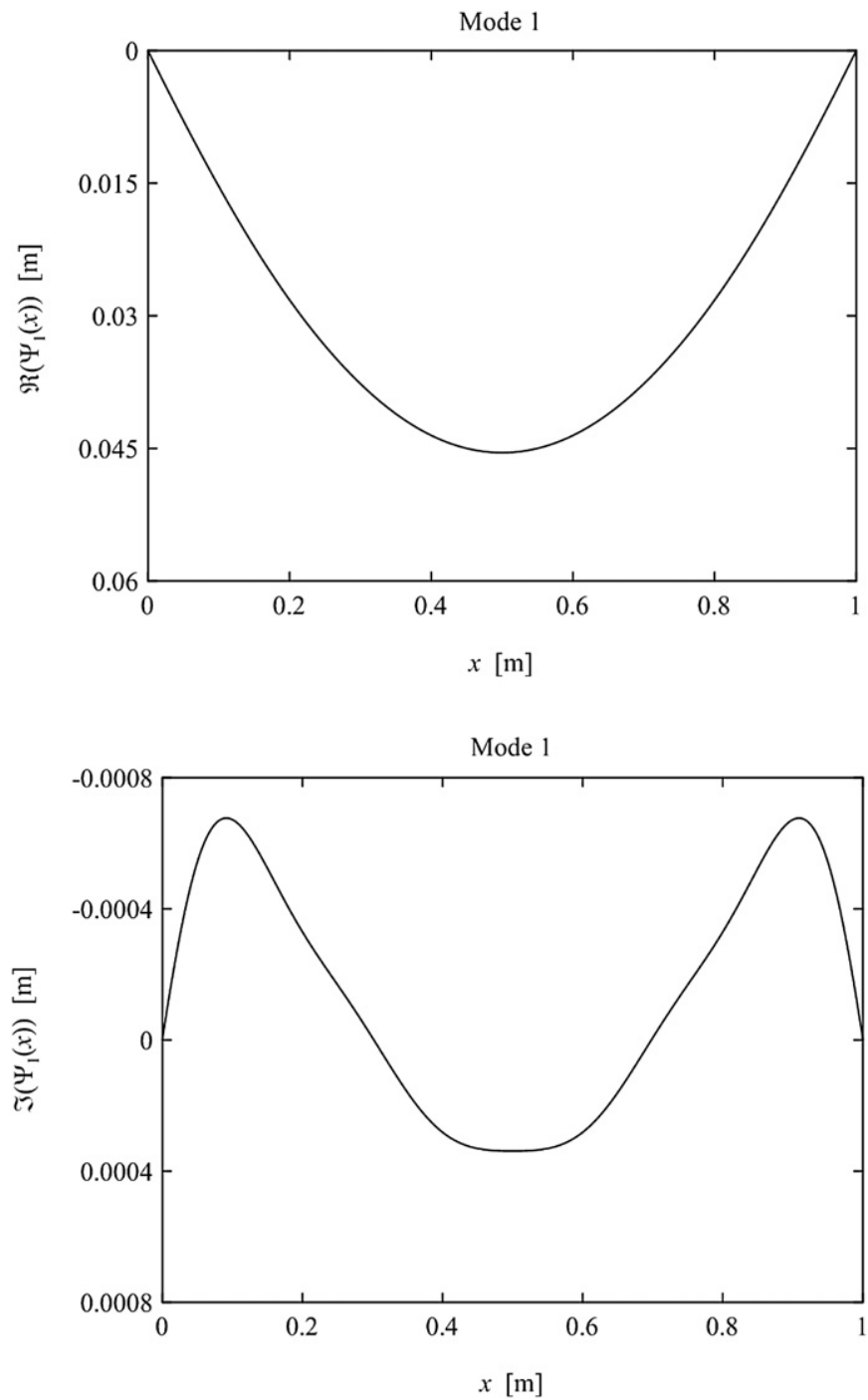

Fig. 7. Fiberglass-polyester composite beam: real and imaginary parts of complex mode 1. the actual microstructure at the fiber-matrix interface (that, however, is virtually impossible due to several, inherent uncertainties) but, consistently with a typical approach of non-local theories, they account for the coupling effects due to the fibers in an average sense.

Specifically, the following numerical values are selected for $C_{r}$ and $l_{r}, C_{d}$ and $l_{d}$ in Eq. (32): $C_{r}=2 \cdot 10^{2} \mathrm{~m}^{-6}$ and $l_{r}=0.5 \mathrm{~m}$ in Eq. (32a); $C_{d}=2 \cdot 10^{-2} \mathrm{~m}^{-6}$ and different values of the internal length $l_{d}$ in Eq. (32b).

In the constitutive equations (5) $\beta_{1}=1$ is selected. As a result of this choice, the non-local solution will tend to the solution obtained by the classical local TM theory, as $l_{r} \rightarrow 0$ and $l_{d} \rightarrow 0$ in $g(x, \xi)$ and $\tilde{g}(x, \xi)$ given by Eq. (32).

The numerical results reported are obtained for $N=800$ intervals in the beam domain. To solve the complex eigenvalue problem (28), $M=10$ undamped modes are retained in the eigenvector expression (29). No significant variations are found in the solution, for $N>800$ and $M>10$.

The first four eigenvalues are reported in Table 2. As the real part of all the eigenvalues is not equal to zero, it is evident that the free vibration response of the beam is damped and decays with time.

Table 3 gives the damping ratio $\zeta_{j}=-\Re\left(s_{j}\right) /\left|s_{j}\right|$ corresponding to the first four eigenvalues in Table 2 . Regardless of the $L / h$ ratio, it is
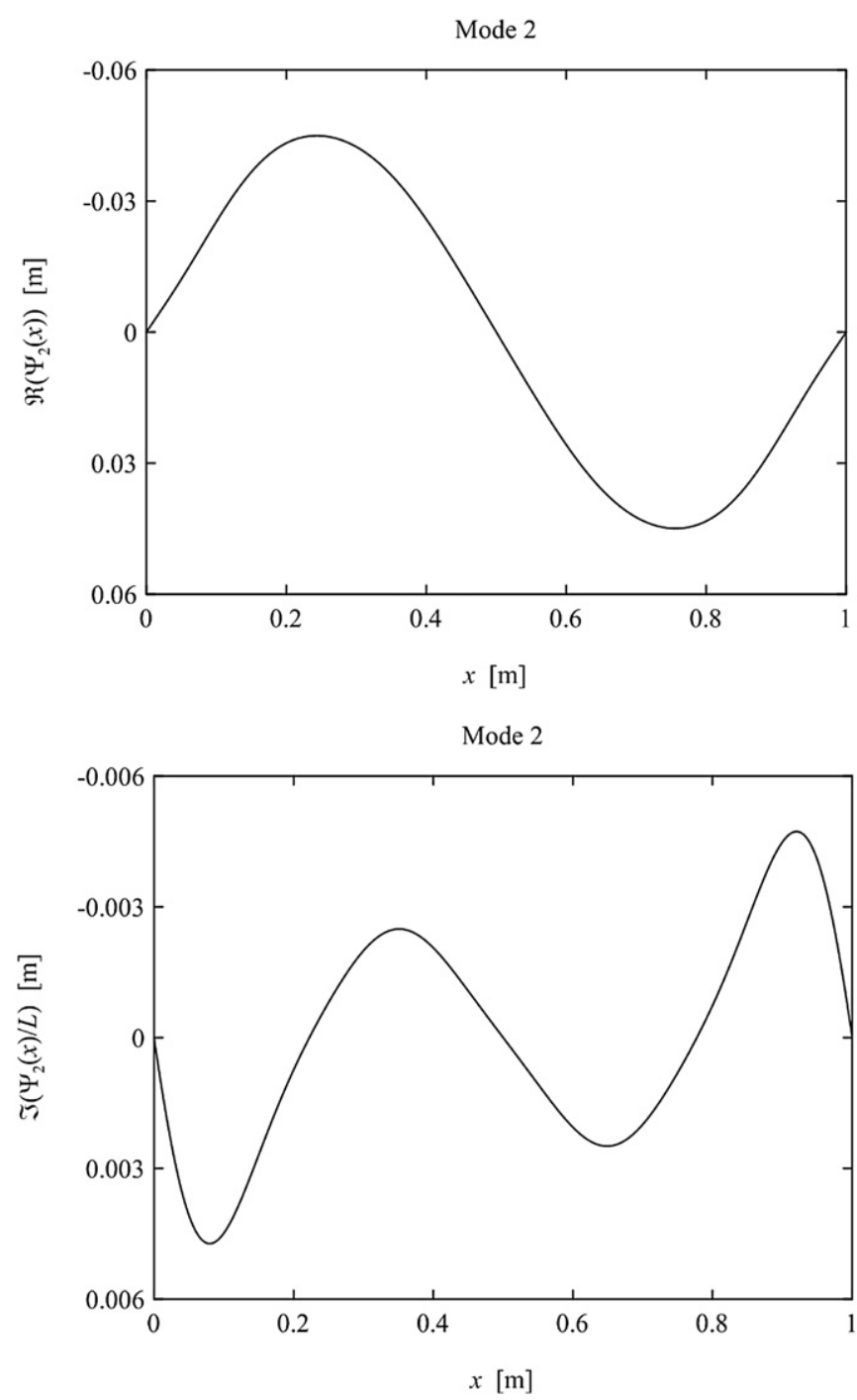

Fig. 8. Fiberglass-polyester composite beam: real and imaginary parts of complex mode 2 . 
seen that the damping ratios increase with the internal length $l_{d}$. In fact the larger is the internal length $l_{d}$, the larger is the amount of interacting beam segments and, consequently, the energy dissipation. Still regardless of the $L / h$ ratio, it is seen that the damping ratio is larger for higher vibration modes. Higher vibration modes involve indeed a larger relative motion between beam segments and, consequently, they are affected by a larger energy dissipation. In this respect, this behavior appears consistent with the results of many experimental tests, where higher damping is encountered in higher vibration modes (Russell, 1992). As concerns the dependence on the $L / h$ ratio, it is noted that the damping ratios are larger as the $L /$ $h$ ratio decreases, i.e. as the height $h$ increases ( $L$ is held constant). This result shall be attributed to the fact that higher values of $h$ determine an increased magnitude of the viscous long-range resultants (as in fact the stiffness $E^{*} I$ is involved in the long-range resultants in Eqs. (22) and (23)) and, therefore, a larger energy dissipation. It shall be finally pointed out that, for the selected parameters, the damping ratios are $<1$ for any value of the internal length $l_{d}$, i.e. the modes are all oscillatory. This appears consistent with typical behaviors of composite structural components. However, it can be readily seen that higher values of $C_{d}$ (i.e. $C_{d}>2 \cdot 10^{-2} \mathrm{~m}^{-6}$ ) would generally lead to damping ratios $=1$, i.e. to non-oscillatory modes, for almost any value of the internal length $l_{d}$.
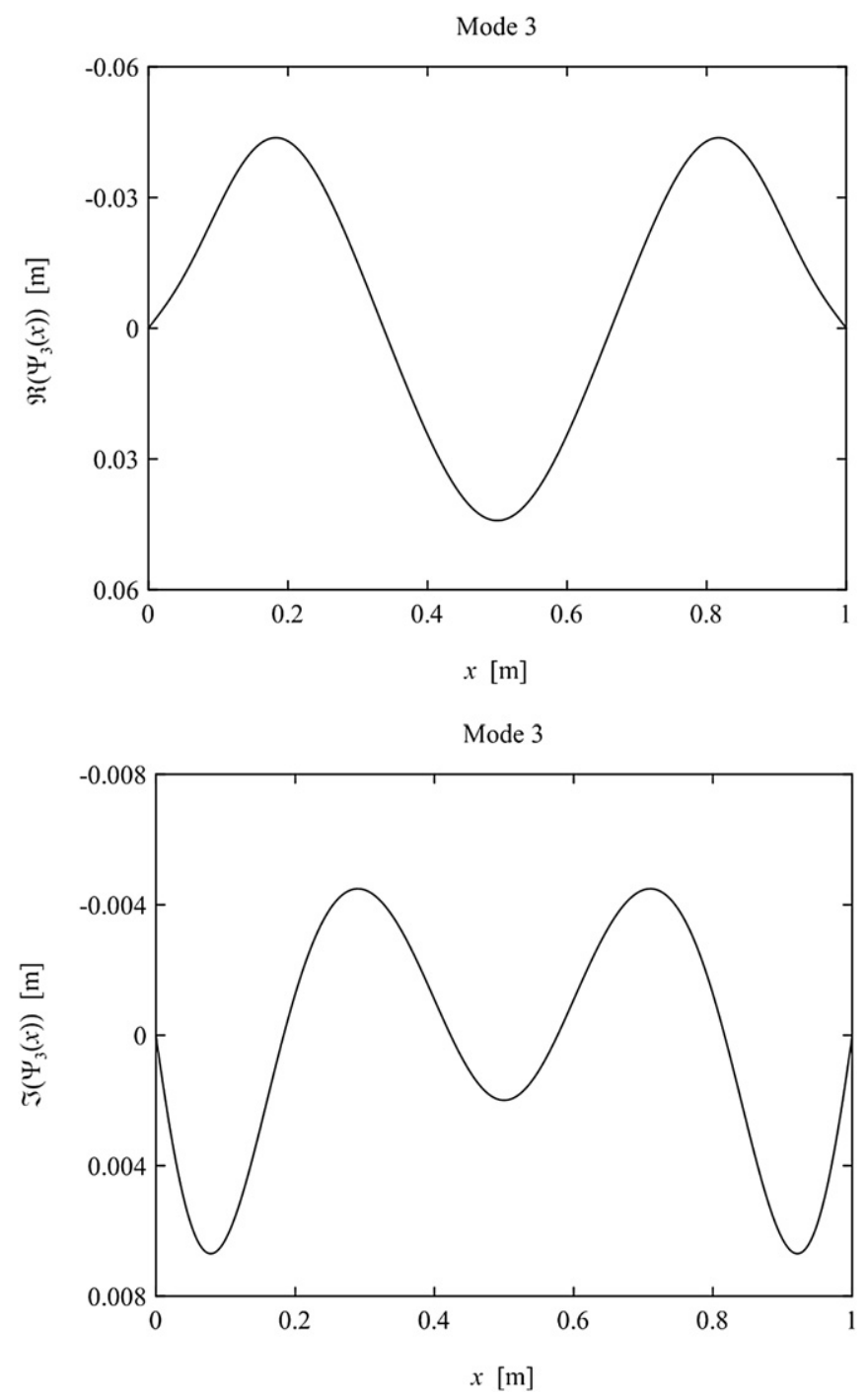

Fig. 9. Fiberglass-polyester composite beam: real and imaginary parts of complex mode 3.
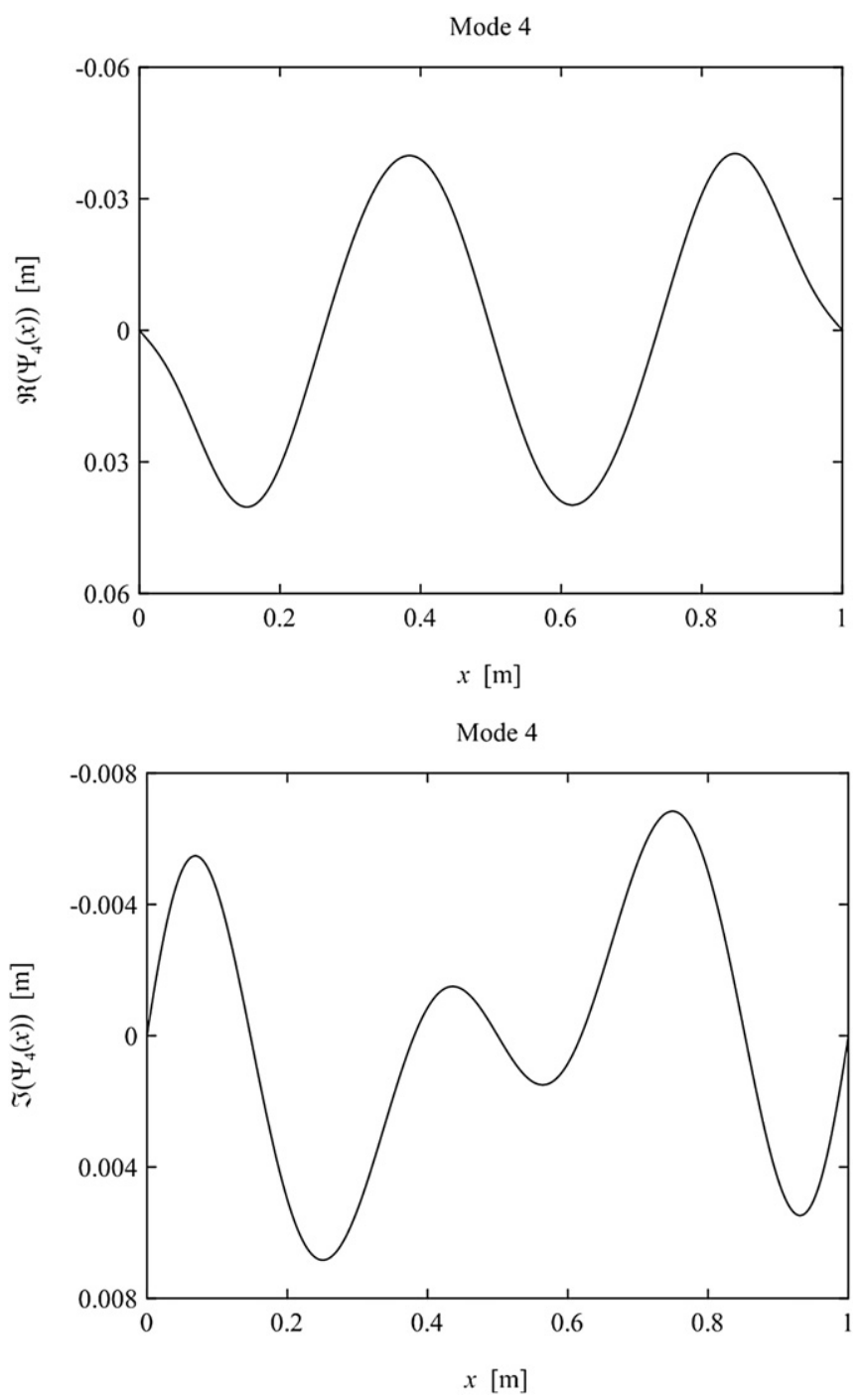

Fig. 10. Fiberglass-polyester composite beam: real and imaginary parts of complex mode 4.

Figs. 7-10 show the real and the imaginary parts of the first four vibration modes of the non-locally damped beam, for the following parameters: $C_{r}=2 \cdot 10^{2} \mathrm{~m}^{-6}, C_{d}=2 \cdot 10^{-2} \mathrm{~m}^{-6}, l_{r}=0.5 \mathrm{~m}$ and $l_{d}=0.06 \mathrm{~m}$ in Eq. (32), $L / h=5$. They are all normalized with respect to the mass matrix. As expected (Friswell et al., 2007), real and imaginary parts reflect the symmetry or the asymmetry of the corresponding local undamped modes.

\section{Concluding remarks}

A non-local TM beam has been presented. As in most common non-local beam theories, the mathematical approach relies on formulating an enriched continuum model of the beam, where non-local effects are accounted for, in an average sense, by introducing non-local terms in the motion equations. Specifically, the non-local terms have been modeled as long-range volume forces/ moments mutually exerted by non-adjacent beam segments, that contribute to the equilibrium of any beam segment along with the classical stress resultants exerted by the adjacent beam segments. Elastic and viscous long-range volume forces/moments have been introduced, to be considered either separately or simultaneously. They are taken as linearly depending on the product of the volumes of the interacting beam segments and on generalized measures of 
their relative motion, through pertinent attenuation functions. As a result, the equilibrium equations of the beam have been built in an integro-differential form, while the mechanical B.C. have been derived in the same form of the classical local theory. Indeed the long-range volume forces/moments are infinitesimals of higher order, that do not contribute to the equilibrium equation at the beam ends. The generalized measures of relative motion have been built based on the pure deformation modes of the TM beam, to ensure that the long-range volume forces/moments are invariant with respect to rigid body motion.

The proposed model lends itself to address different sources of non-local effects. If elastic long-range volume forces/moments only are considered, it can be seen as a generalization of a non-local bar model already developed by the authors (Di Paola et al., 2009, 2011b; Failla et al., 2010), with typical applications of alternative non-local beam theories, as for instance to capture small-size effects in micro- or nano-beams. If either viscous or elastic and viscous (i.e. viscoelastic) long-range volume forces/moments are considered, it can be seen as a generalization of the non-local viscous damping model by Russell (1992) to address, for instance, non-local damping effects in fiber-reinforced composite beams.

In the proposed model a certain number of functions/parameters are involved, which affect the non-local terms, i.e. the attenuation functions governing axial, bending and shear behavior and pertinent parameters (in this paper, the exponential functions (31)-(32) and parameters $C_{r}$ and $l_{r}, C_{d}$ and $l_{d}$ ), as well as the local terms (see $\beta_{1}$ in Eq. (5)).

The mathematical form of the attenuation functions, the related non-local parameters as well as parameter $\beta_{1}$ in Eq. (5), shall be generally determined via an optimization procedure, where the theoretical model is fitted to experimental results, on static or dynamic response. It is important to note that a similar procedure is not characteristic of the proposed model only, but is typical of all classical non-local theories involving an enriched continuum with additional non-local terms. Depending on the formulation, the non-local terms always involve a number of unknown parameters that only experimental evidence may allow to select. For instance, examples of enriched continua with five additional parameters for non-local terms exist in the literature (Lam et al., 2003; Mindlin, 1965). Obviously, any optimization procedure to fit experimental evidence shall be generally preceded by numerical simulations, that may serve to determine the expected order of magnitude of the non-local terms, with respect to the local ones. An example of a fitting procedure has been proposed by Banks and Inman (1991).

As specifically concerns the selection of the attenuation functions when elastic and viscous long-range resultants are simultaneously introduced in the model, i.e., when viscoelastic long-range resultants are considered, for generality they have been allowed to be taken as independent functions. This option has been considered in view of ensuring that, when fitted to experimental results, the proposed model can be as much versatile as possible. In practice, it may be thought that they have the same mathematical form, but different parameters (see also comments in Section 5.2).

In the numerical applications, parameters and attenuation functions have been set on a theoretical basis, to enhance non-local effects. The undamped free vibrations of an epoxy micro-beam and the damped free vibrations of a fiberglass-polyester beam have been addressed, the latter when elastic and viscous long-range resultants are simultaneously included in the model. This case corresponds to a Kelvin-Voigt viscoelastic modeling of the dissipation mechanism at the fiber-matrix interface. From a qualitative point of view, the results have been found consistent with typical behaviors emerged by experimental tests, such as a stiffening effect of the undamped vibration modes due to small-size effects in micro-beams (Lam et al., 2003; McFarland and Colton,
2005), and an increase of damping in the higher vibration modes of fiber-reinforced composite beams (Russell, 1992). The proposed model appears however sufficiently versatile and potentially capable of predicting different behaviors, as they should be suggested by experimental evidence. For instance an appropriate selection of parameter $\beta_{1}(<1)$ could be made whereas a softening with respect to classical local solution is expected, as a result of non-local effects (Di Paola et al., 2010b, 2011a). Potential generalizations to alternative damping mechanisms, such as for instance a fractional viscoelastic damping, are feasible. Pertinent results will be presented in a self-contained study.

A final remark concerns the solution of the motion equations. The proposed model lends itself to numerical solutions, by a finite element modeling or by a Galerkin series expansion, computationally more efficient than the finite difference solution built in this paper. In this respect, however, it shall be pointed out that the finite difference solution generally requires a relatively high number of intervals when an accurate description of the high frequency modes is requested, and that the computational effort involved drastically reduces if only the first modes are of interest.

\section{Appendix A}

It is of interest to compare the non-local TM beam proposed in this paper to the non-local TM beam previously built by Di Paola et al. (2011a, 2012), in context with the general formulation of a non-local 3D continuum.

Both are conceived on the key assumption that non-local effects can be modeled as long-range volume forces, see Eq. (10) of the paper by Di Paola et al. (2011a) or volume forces/moments, see Eq. (6) of this paper. Both are displacement based, that is non-local effects are assumed to stem from the relative displacements (and rotations, in this paper) between non-adjacent interacting volume elements. However some relevant differences exist, as explained in the following.

\section{A.1. Non-local TM beam by Di Paola et al. (2011a, 2012)}

In the non-local TM beam built by Di Paola et al. (2011a, 2012), the long-range forces have been modeled as elementary volume forces mutually exerted by non-adjacent elementary volume elements. These forces are central forces, i.e. they are directed along the line joining the positions of the interacting volume elements in the original configuration. Further, they are built as linearly depending on the product of the interacting volume elements and on their relative displacement (as measured along the line joining the corresponding positions in the original configuration), through an appropriate attenuation function. Based on the kinematic model of the TM theory, the principle of virtual work has been used to derive long-range resultants per unit length (forces and moments), in the form given by Eq. (34) of the paper by Di Paola et al. (2011a), and here reported for completeness:

$$
\begin{aligned}
& R_{1}(x)=\int_{0}^{L}\left\{I_{1}(x, \xi)[u(\xi)-u(x)]-I_{7}(x, \xi) \varphi(\xi)+I_{8}(x, \xi) \varphi(x)\right. \\
& \left.+I_{9}(x, \xi)[v(\xi)-v(x)]\right\} \mathrm{d} \xi ; \\
& R_{2}(x)=\int_{0}^{L}\left\{I_{9}(x, \xi)[u(\xi)-u(x)]-I_{2}(x, \xi) \varphi(\xi)+I_{3}(x, \xi) \varphi(x)\right. \\
& \left.+I_{4}(x, \xi)[v(\xi)-v(x)]\right\} \mathrm{d} \xi ;
\end{aligned}
$$




$$
\begin{aligned}
R_{3}(x)=\int_{0}^{L}\left\{-I_{8}(x, \xi)[u(\xi)-u(x)]+I_{5}(x, \xi) \varphi(\xi)-I_{6}(x, \xi) \varphi(x)\right. \\
\left.\quad-I_{3}(x, \xi)[v(\xi)-v(x)]\right\} \mathrm{d} \xi
\end{aligned}
$$

In Eq. (A.1), $R_{1}(x), R_{2}(x)$ and $R_{3}(x)$ are the long-range axial force, transverse force and moment per unit length, $I_{i}(x, \xi)$ for $(i=1,2, \ldots, 9)$ are integrals depending on the cross section (note that $I_{7}(x, \xi)=I_{8}(x, \xi)$ always holds, for any cross section $)$.

A relevant feature in Eq. (A.1) is that, in a beam of arbitrary cross section, a long-range axial force $R_{1}(x)$, see Eq. (A.1a), may arise not only due to a relative axial displacement but, also, due to a relative transverse displacement or a relative rotation. Such a coupling is not encountered, instead, if the cross section has a double symmetry, being in this case $I_{7}(x, \xi)=I_{8}(x, \xi)=I_{9}(x, \xi)=0$. An explanation of this result may be found on a mechanical basis, as explained in the following.

In the TM beam by Di Paola et al. (2011a, 2012), it can be thought that the long-range elementary volume forces mutually exerted by two elementary volume elements are yielded by an elementary truss that connects them in the original configuration. As a result, a truss-like connection is established between non-adjacent beam segments, where each elementary truss corresponding to a couple of interacting volume elements may undergo either traction or compression, depending on the relative displacement/rotation between the beam segments. It can be seen that, if the cross section features a double symmetry, no total long-range axial force arises on a beam segment at $x$, as a result of a relative transverse displacement with respect to a beam segment at $\xi \neq x$. In fact the contribution to the total long-range axial force given by any couple $\mathrm{P}-\mathrm{Q}$ of interacting volume elements is balanced by the contributions given by other couples of interacting volume elements that, along with the couple $\mathrm{P}-\mathrm{Q}$, form a symmetric truss. An example is shown in Fig. 11: in the beam with an I section, where the beam segments at $x$ and $\xi$ undergo the relative transverse displacement $v(\xi)-v(x)$, the contribution to the total long-range force given by the couple $\mathrm{P}-\mathrm{Q}$ is balanced by the contributions given by the couples $\mathrm{P}_{1}-\mathrm{Q}, \mathrm{P}_{2}-\mathrm{Q}_{1}, \mathrm{P}_{3}-\mathrm{Q}_{1}$ : the four couples form indeed a symmetric truss where the axial component of the force in truss $n .1$ is balanced by the axial component of the force in truss n.2. On the contrary it can be seen that, if the cross section features a single symmetry, a total long-range axial force does arise on a beam segment at $x$, as a result of a relative transverse displacement with respect to a beam segment at $\xi \neq x$ due to the fact that, in general, the contribution given by a couple $\mathrm{P}-\mathrm{Q}$ of interacting volume elements cannot be balanced by the contributions given by other couples that may form, along with the couple $\mathrm{P}-\mathrm{Q}$, a symmetric truss. For instance in the beam with a T section shown in Fig. 11, it is evident that the axial component of the force in truss n.1 cannot be balanced by the axial component of any of the other elementary trusses between the beam segments at $x$ and $\xi$. The same statements hold true also whereas two beam segments undergo a relative rotation.

A further, important consideration on Eq. (A.1) is that, even if the cross section has a double symmetry, a relative transverse



Truss n. 1

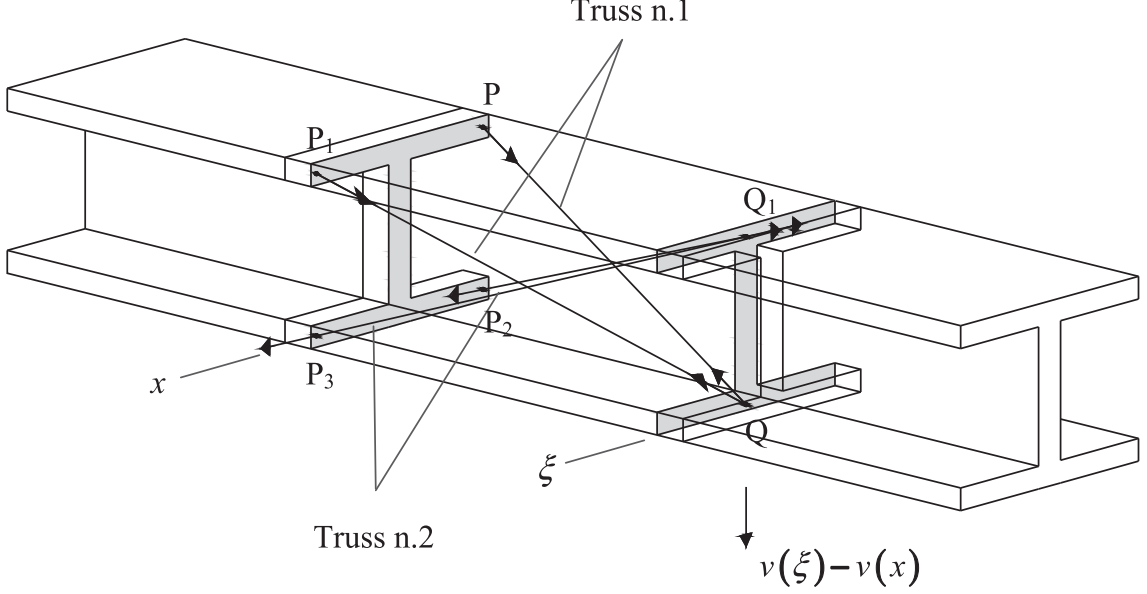

Fig. 11. Mechanics of the non-local beam by Di Paola et al. (2011a) for single- and double-symmetric sections. 
displacement always determines long-range transverse forces (see Eq. (A1.b)). This means that the beam model by Di Paola et al. (2011a, 2012) is not suitable for those applications where it can be assumed, for instance, that non-local effects result only in longrange moments but not in long-range transverse forces, as it may be the case in composite beams where long fibers passing through a material matrix are placed only at the upper and lower surface of the beam (Russell, 1992).

\section{A.2. Proposed non-local TM beam}

In the non-local TM beam proposed in this paper, consistently with typical engineering beam theories where the equilibrium of a beam segment is set in a weak sense based on the stress resultants on the cross section, the non-local effects have not been modeled as elementary forces (Di Paola et al., 2011a, 2012), but they have been given an analytical form in terms of long-range volume forces/moments exchanged between non-adjacent beam segments. As shown in Figs. 3 and 4, from a mechanical point of view this can be considered as equivalent to assuming a spring-like connection between non-adjacent beam segments where pure axial, pure bending and pure shear long-range springs can be separately accounted for. The model of this paper is then suitable for those applications where it can be assumed that non-local effects result only in long-range moments but not in long-range transverse forces (Russell, 1992).

It shall be also pointed out that, in the beam model built by $\mathrm{Di}$ Paola et al. (2011a, 2012), the integrals $I_{i}(x, \xi)$ for $(i=1,2, \ldots, 9)$ have to be computed numerically, for any couple $(x, \xi)$ singled out in the beam axis, discretized according to the finite difference method. Depending on the applications at hand, the computational effort may be significant. The computational effort required by the beam model of this paper is drastically lower, as no numerical integration is required to evaluate the non-local stiffness terms.

\section{References}

Aifantis, E.C., 1984. On the microstructural origin of certain inelastic models. J. Eng. Mater - Trans. ASME 106, 326-330.

Aifantis, E.C., 1999. Gradient deformation models at nano, micro, and macroscales. J. Eng. Mater. Technol. - Trans. ASME 121, 189-202.

Altan, B.S., 1989. Uniqueness of the initial-value problems in nonlocal elastic solids. Int. J. Solids Struct. 25, 1271-1278.

Askes, H., Metrikine, A.V., 2002. One-dimensional dynamically consistent gradient elasticity models derived from a discrete microstructure. Part 2: static and dynamic response. Eur. J. Mech. A/Solids 21, 573-588.

Aydogdu, M., 2009. A general nonlocal beam theory: its application to nanobeam bending, buckling and vibration. Phys. E 41, 1651-1655.

Banks, H.T., Inman, D.J., 1991. On damping mechanism in beams. J. Appl. Mech. 58 716-723.

Banks, H.T., Wang, Y., Inman, D.J., 1994. Bending and shear damping in beams: frequency domain estimation techniques. J. Vib. Acoust. 116, 188-197.

Campbell, F.C., 2010. Structural Composite Materials. ASM International, Materials Park

Chang, C.S., Askes, H., Sluys, L.J., 2002. Higher-order strain/higher-order stress gradient models derived from a discrete microstructure, with application to fracture. Eng. Fract. Mech. 69, 1907-1924.

Di Paola, M., Failla, G., Zingales, M., 2009. Physically-based approach to the mechanics of strong non-local linear elasticity theory. J. Elast. 97, 103-130.

Di Paola, M., Pirrotta, A., Zingales, M., 2010a. Mechanically-based approach to nonlocal elasticity: variational principles. Int. J. Solids Struct. 47, 539-548.

Di Paola, M., Failla, G., Zingales, M., 2010b. The mechanically-based approach to 3D non-local linear elasticity theory: long-range central interactions. Int. J. Solids Struct. 47, 2347-2358.

Di Paola, M., Failla, G., Sofi, A., Zingales, M., 2011a. A mechanically based approach to non-local beam theories. Int. J. Mech. Sci. 53, 676-687.

Di Paola, M., Sofi, A., Zingales, M., 2011b. Stochastic analysis of one-dimensional heterogeneous solids with long-range interactions. Int. J. Multiscale Comput. Eng. 9 (4), 379-394.

Di Paola, M., Failla, G., Sofi, A., Zingales, M., 2012. On the vibrations of a mechanically based non-local beam model. Comput. Mater. Sci. 64, 278-282.
Eringen, A.C., 1972. Linear theory of nonlocal elasticity and dispersion of plane waves. Int. J. Eng. Sci. 10, 425-435.

Eringen, A.C., 1983. On differential equations of nonlocal elasticity and solutions of screw dislocation and surface waves. J. Appl. Phys. 54, 4703-4710.

Eringen, A.C., 1987. Theory of nonlocal elasticity and some applications. Res. Mech. 21, 313-342.

Failla, G., Santini, A., Zingales, M., 2010. Solution strategies for 1D elastic continuum with long-range interactions: smooth and fractional decay. Mech. Res. Commun. 37, 13-21.

Failla, G., Santini, A., Zingales, M., 2013. A non-local two-dimensional foundation model. Arch. Appl. Mech. 83, 253-272.

Fisher, F.T., Brinson, L.C., 2001. Viscoelastic interphases in polymer-matrix composites: theoretical models and finite-element analysis. Compos. Sci. Technol. $61,731-748$.

Flugge, W., 1975. Viscoelasticity, second revised ed. Springer-Verlag, Berlin.

Friswell, M.I., Adhikari, S., Lei, Y., 2007. Non-local finite element analysis of damped beams. Int. J. Solids Struct. 44, 7564-7576.

Fuchs, M.B., 1991. Unimodal beam elements. Int. J. Solids Struct. 27 (5), 533-545.

Fuchs, M.B., 1997. Unimodal formulation of the analysis and design problems for framed structures. Comput. Struct. 63 (4), 739-747.

Gosz, M., Moran, B., Achenbach, J.D., 1991. Effect of a viscoelastic interface on the transverse behavior of fiber-reinforced composites. Int. J. Solids Struct. 27 (14), 1757-1771.

Humar, J.L., 2002. Dynamics of Structures, second ed. Swets \& Zeitlinger B.V., Lisse.

Kong, S., Zhou, S., Nie, Z., Wang, K., 2008. The size-dependent natural frequency of Bernoulli-Euler micro-beams. Int. J. Eng. Sci. 46, 427-437.

Lakes, R.S., 1991. Experimental micro mechanics methods for conventional and negative Poisson's ratio cellular solids as Cosserat continua. J. Eng. Mater. Technol. 113, 148-155.

Lam, D.C.C., Yang, F., Chong, A.C.M., Wang, J., Tong, P., 2003. Experiments and theory in strain gradient elasticity. J. Mech. Phys. Solids 51, 1477-1508.

Lei, Y., Friswell, M.I., Adhikari, S., 2006a. A Galerkin method for distributed systems with non-local damping. Int. J. Solids Struct. 43, 3381-3400.

Lei, Y., Friswell, M.I., Adhikari, S., 2006b. The analysis of distributed systems with nonlocal damping. In: Clark, W.W., Ahmadian, M., Lumsdaine, A. (Eds.), Smart Structures and Materials 2006: Damping and Isolation. Proc. SPIE, pp. 61690V1-61690V8.

Lu, P., Lee, H.P., Lu, C., Zhang, P.Q., 2007. Application of nonlocal beam models for carbon nanotubes. Int. J. Solids Struct. 44, 5289-5300.

Matzenmiller, A., Gerlach, S., 2004. Micromechanical modeling of viscoelastic composites with compliant fiber-matrix bonding. Comput. Mater. Sci. 29, 283-300.

McFarland, A.W., Colton, J.S., 2005. Role of material microstructure in plate stiffness with relevance to microcantilever sensors. J. Micromech. Microeng. 15, 10601067.

Metrikine, A.V., Askes, H., 2002. One-dimensional dynamically consistent gradient elasticity models derived from a discrete microstructure. Part 1: generic formulation. Eur. J. Mech. A/Solids 21, 555-572.

Mindlin, R.D., 1965. Second gradient of strain and surface tension in linear elasticity. Int. J. Solids Struct. 1, 417-438

Murmu, T., Pradhan, S.C., 2009. Small-scale effect on the vibration of nonuniform nanocantilever based on nonlocal elasticity theory. Phys. E 41, 1451-1456.

Polizzotto, C., 2001. Non local elasticity and related variational principles. Int. J. Solids Struct. 38, 7359-7380.

Poole, W.J., Ashby, M.F., Fleck, N.A., 1996. Micro-hardness of annealed and workhardened copper polycrystals. Scr. Mater. 34 (4), 559-564.

Qian, D., Wagner, G.J., Liu, W.K., Yu, M.-F., Ruoff, R.S., 2002. Mechanics of carbon nanotubes. Appl. Mech. Rev. 55 (6), 495-533.

Reddy, J.N., 2007. Nonlocal theories for bending, buckling and vibration of beams. Int. J. Eng. Sci. 45, 288-307.

Russell, D.L., 1992. On mathematical models for the elastic beam with frequencyproportional damping. In: Banks, H.T. (Ed.), Control and Estimation in Distributed Parameter Systems. SIAM, Philadelphia, PA, pp. 125-169.

Silling, S.A., 2000. Reformulation of elasticity theory for discontinuities and longrange forces. J. Mech. Phys. Solids 48, 175-209.

Silling, S.A., Zimmermann, M., Abeyaratne, R., 2003. Deformation of a peridynamic bar. J. Elast. 73, 173-190.

Sorrentino, S., Marchesiello, S., Piombo, B.A.D., 2003. A new analytical technique for vibration analysis of non-proportionally damped beams. J. Sound Vib. 265 (4), 765-782.

Tang, P.Y., 1983. Interpretation of bend strength increase of graphite by the couple stress theory. Comput. Struct. 16, 45-49.

Wang, L.F., Hu, H.Y., 2005. Flexural wave propagation in single-walled carbon nanotube. Phys. Rev. B 71, 195412-195418.

Wang, B., Zhao, J., Zhou, S., 2010. A micro scale Timoshenko beam model based on strain gradient elasticity theory. Eur. J. Mech. A/Solids 29, 591-599.

$\mathrm{Xu}, \mathrm{M} ., 2006$. Free transverse vibrations of nano-to-micron scale beams. Proc. R. Soc. A 462, 2977-2995.

Zhang, Y.Y., Wang, C.M., Challamel, N., 2010. Bending, buckling and vibration of micro/nanobeams by hybrid nonlocal beam model. J. Eng. Mech. 136 (5), 562-574.

Zhao, X.C., Lei, Y.J., Zhou, J.P., 2008. Strain analysis of nonlocal viscoelastic Kelvin bar in tension. Appl. Math. Mech. 29 (1), 67-74. 